\title{
Hopf Bifurcation and Global Periodic Solutions in a Predator-Prey System with Michaelis-Menten Type Functional Response and Two Delays
}

\author{
Yunxian Dai, ${ }^{1}$ Yiping Lin, ${ }^{1}$ and Huitao Zhao $^{1,2}$ \\ ${ }^{1}$ Department of Applied Mathematics, Kunming University of Science and Technology, Kunming, Yunnan 650093, China \\ ${ }^{2}$ Department of Mathematics and Information Science, Zhoukou Normal University, Zhoukou, Henan 466001, China
}

Correspondence should be addressed to Yiping Lin; linyiping689@163.com

Received 27 November 2013; Accepted 31 March 2014; Published 11 May 2014

Academic Editor: Yuming Chen

Copyright (C) 2014 Yunxian Dai et al. This is an open access article distributed under the Creative Commons Attribution License, which permits unrestricted use, distribution, and reproduction in any medium, provided the original work is properly cited.

We consider a predator-prey system with Michaelis-Menten type functional response and two delays. We focus on the case with two unequal and non-zero delays present in the model, study the local stability of the equilibria and the existence of Hopf bifurcation, and then obtain explicit formulas to determine the properties of Hopf bifurcation by using the normal form method and center manifold theorem. Special attention is paid to the global continuation of local Hopf bifurcation when the delays $\tau_{1} \neq \tau_{2}$.

\section{Introduction}

In [1], $\mathrm{Xu}$ and Chaplain studied the following delayed predator-prey model with Michaelis-Menten type functional response:

$$
\begin{aligned}
\frac{d x_{1}}{d t}=x_{1}(t) & {\left[a_{1}-a_{11} x_{1}\left(t-\tau_{11}\right)-\frac{a_{12} x_{2}(t)}{m_{1}+x_{1}(t)}\right], } \\
\frac{d x_{2}}{d t}=x_{2}(t) & {\left[-a_{2}+\frac{a_{21} x_{1}\left(t-\tau_{21}\right)}{m_{1}+x_{1}\left(t-\tau_{21}\right)}\right.} \\
& \left.\quad-a_{22} x_{2}\left(t-\tau_{22}\right)-\frac{a_{23} x_{3}(t)}{m_{2}+x_{2}(t)}\right], \\
\frac{d x_{3}}{d t}=x_{3}(t) & {\left[-a_{3}+\frac{a_{32} x_{2}\left(t-\tau_{32}\right)}{m_{2}+x_{2}\left(t-\tau_{32}\right)}-a_{33} x_{3}\left(t-\tau_{33}\right)\right], }
\end{aligned}
$$

with initial conditions

$$
x_{i}(t)=\phi_{i}(t), \quad t \in[-\tau, 0], \quad \phi_{i}(0)>0, \quad i=1,2,3,
$$

where $x_{1}(t), x_{2}(t)$, and $x_{3}(t)$ denote the densities of the prey, predator, and top predator population, respectively. $a_{i}, a_{i j}(i, j=1,2,3)$ are positive constants. $\tau_{11}, \tau_{21}, \tau_{22}, \tau_{32}$, and $\tau_{33}$ are nonnegative constants. $\tau_{11}, \tau_{22}, \tau_{33}$ denote the delay in the negative feedback of the prey, predator, and top predator crowding, respectively. $\tau_{21}, \tau_{32}$, are constant delays due to gestation; that is, mature adult predators can only contribute to the production of predator biomass. $\tau=$ $\max \left\{\tau_{11}, \tau_{21}, \tau_{22}, \tau_{32}, \tau_{33}\right\} . \phi_{i}(t)(i=1,2,3)$ are continuous bounded functions in the interval $[-\tau, 0]$. The authors proved that the system is uniformly persistent under some appropriate conditions. By means of constructing suitable Lyapunov functional, sufficient conditions are derived for the global asymptotic stability of the positive equilibrium of the system.

Time delays of one type or another have been incorporated into systems by many researchers since a time delay could cause a stable equilibrium to become unstable and fluctuation. In [2-12], authors showed effects of two delays on dynamical behaviors of system.

It is well known that periodic solutions can arise through the Hopf bifurcation in delay differential equations. However, these periodic solutions bifurcating from Hopf bifurcations are generally local. Under some circumstances, periodic solutions exist when the parameter is far away from the critical value. Therefore, global existence of Hopf bifurcation 
is a more interesting and difficult topic. A great deal of research has been devoted to the topics [13-21]. In this paper, let $\tau_{11}=\tau_{22}=\tau_{33}=0, \tau_{21}=\tau_{1}, \tau_{32}=\tau_{2}$ in (1); we consider Hopf bifurcation and global periodic solutions of the following system with two unequal and nonzero delays:

$$
\begin{aligned}
& \frac{d x_{1}}{d t}=x_{1}(t) {\left[a_{1}-a_{11} x_{1}(t)-\frac{a_{12} x_{2}(t)}{m_{1}+x_{1}(t)}\right], } \\
& \frac{d x_{2}}{d t}=x_{2}(t)[-a_{2}+\frac{a_{21} x_{1}\left(t-\tau_{1}\right)}{m_{1}+x_{1}\left(t-\tau_{1}\right)} \\
&\left.-a_{22} x_{2}(t)-\frac{a_{23} x_{3}(t)}{m_{2}+x_{2}(t)}\right], \\
& \frac{d x_{3}}{d t}=x_{3}(t)\left[-a_{3}+\frac{a_{32} x_{2}\left(t-\tau_{2}\right)}{m_{2}+x_{2}\left(t-\tau_{2}\right)}-a_{33} x_{3}(t)\right],
\end{aligned}
$$

with initial conditions

$$
\begin{aligned}
x_{i}(t)=\phi_{i}(t), \quad t \in[-\tau, 0], \quad \phi_{i}(0)>0, \\
i=1,2,3 ; \quad \tau=\max \left\{\tau_{1}, \tau_{2}\right\} .
\end{aligned}
$$

Our goal is to investigate the possible stability switches of the positive equilibrium and stability of periodic orbits arising due to a Hopf bifurcation when one of the delays is treated as a bifurcation parameter. Special attention is paid to the global continuation of local Hopf bifurcation when the delays $\tau_{1} \neq \tau_{2}$.

This paper is organized as follows. In Section 2, by analyzing the characteristic equation of the linearized system of system (3) at positive equilibrium, the sufficient conditions ensuring the local stability of the positive equilibrium and the existence of Hopf bifurcation are obtained [22]. Some explicit formulas determining the direction and stability of periodic solutions bifurcating from Hopf bifurcations are demonstrated by applying the normal form method and center manifold theory due to Hassard et al. [23] in Section 3. In Section 4, we consider the global existence of these bifurcating periodic solutions [24] with two different delays. Some numerical simulation results are included in Section 5 .

\section{Stability of the Positive Equilibrium and Local Hopf Bifurcations}

In this section, we first study the existence and local stability of the positive equilibrium and then investigate the effect of delay and the conditions for existence of Hopf bifurcations. (3):

There are at most four nonnegative equilibria for system

$$
\begin{aligned}
& E_{1}=(0,0,0), \quad E_{2}=\left(\frac{a_{1}}{a_{11}}, 0,0\right), \\
& E_{3}=\left(\tilde{x}_{1}, \tilde{x}_{2}, 0\right), \quad E_{*}=\left(x_{1}^{*}, x_{2}^{*}, x_{3}^{*}\right),
\end{aligned}
$$

where $\left(\tilde{x}_{1}, \tilde{x}_{2}, 0\right)$ and $\left(x_{1}^{*}, x_{2}^{*}, x_{3}^{*}\right)$ satisfy

$$
\begin{gathered}
a_{1}-a_{11} \tilde{x}_{1}-\frac{a_{12} \tilde{x}_{2}}{m_{1}+\tilde{x}_{1}}=0, \\
-a_{2}+\frac{a_{21} \tilde{x}_{1}}{m_{1}+\tilde{x}_{1}}-a_{22} \tilde{x}_{2}=0 \\
a_{1}-a_{11} x_{1}^{*}(t)-\frac{a_{12} x_{2}^{*}(t)}{m_{1}+x_{1}^{*}(t)}=0 \\
-a_{2}+\frac{a_{21} x_{1}^{*}(t)}{m_{1}+x_{1}^{*}(t)}-a_{22} x_{2}^{*}(t)-\frac{a_{23} x_{3}^{*}(t)}{m_{2}+x_{2}^{*}(t)}=0, \\
-a_{3}+\frac{a_{32} x_{2}^{*}(t)}{m_{2}+x_{2}^{*}(t)}-a_{33} x_{3}^{*}(t)=0,
\end{gathered}
$$

where $E_{3}$ is a nonnegative equilibrium point if there is a positive solution of (6), and $E_{*}$ is a nonnegative equilibrium point if there is a positive solution of (7).

Let

$$
\begin{aligned}
& \left(H_{1}\right) a_{1}\left(a_{21}-a_{2}\right)>m_{1} a_{2} a_{11} ; \\
& \left(H_{2}\right)\left(a_{32}-a_{3}\right)\left[a_{1}\left(a_{21}-a_{2}\right)-m_{1} a_{2}\right]-m_{2} a_{3} a_{22}\left(a_{1}+m_{1} a_{11}\right)>0 ; \\
& \left(H_{3}\right) \widetilde{x}_{2}\left(a_{32}-a_{3}\right)-m_{2} a_{3}>0 ; \\
& \left(H_{4}\right) a_{22}\left(a_{11}-\left(a_{1} / m_{1}\right)\right)-\left(a_{12} a_{21} / m_{1}^{2}\right)>0 .
\end{aligned}
$$

From $[1,25]$, we know that if $\left(H_{1}\right),\left(H_{2}\right),\left(H_{3}\right)$, and $\left(H_{4}\right)$ hold, $E_{3}$ and $E_{*}$ always exist as nonnegative equilibria.

Let $E=\left(x_{10}, x_{20}, x_{30}\right)$ be the arbitrary equilibrium point, and let $\bar{x}_{1}(t)=x_{1}(t)-x_{10}, \bar{x}_{2}(t)=x_{2}(t)-x_{20}, \bar{x}_{3}(t)=$ $x_{3}(t)-x_{30}$; still denote $\bar{x}_{1}(t), \bar{x}_{2}(t), \bar{x}_{3}(t)$ by $x_{1}(t), x_{2}(t), x_{3}(t)$, respectively; then the linearized system of the corresponding equations at $E$ is as follows:

$$
\dot{u}(t)=B u(t)+C u\left(t-\tau_{1}\right)+D u\left(t-\tau_{2}\right),
$$

where

$$
\begin{aligned}
& u(t)=\left(x_{1}(t), x_{2}(t), x_{3}(t)\right)^{T}, \\
& B=\left(b_{i j}\right)_{3 \times 3}, \quad C=\left(c_{i j}\right)_{3 \times 3}, \quad D=\left(d_{i j}\right)_{3 \times 3} ; \\
& b_{11}=a_{1}-2 a_{11} x_{10}-\frac{a_{12} m_{1} x_{20}}{\left(m_{1}+x_{10}\right)^{2}}, \quad b_{12}=-\frac{a_{12} x_{10}}{m_{1}+x_{10}}, \\
& b_{22}=-a_{2}+\frac{a_{21} x_{10}}{m_{1}+x_{10}}-2 a_{22} x_{20}-\frac{a_{23} m_{2} x_{30}}{\left(m_{2}+x_{20}\right)^{2}}, \\
& b_{23}=-\frac{a_{23} x_{20}}{m_{2}+x_{20}}, \quad b_{33}=-a_{3}+\frac{a_{32} x_{20}}{m_{2}+x_{20}}-2 a_{33} x_{30} ; \\
& c_{21}=\frac{a_{21} m_{1} x_{20}}{\left(m_{1}+x_{10}\right)^{2}}, \quad d_{32}=\frac{a_{32} m_{2} x_{30}}{\left(m_{2}+x_{20}\right)^{2}}
\end{aligned}
$$

all the others of $b_{i j}, c_{i j}$, and $d_{i j}$ are 0 .

The characteristic equation for system (8) is

$$
\lambda^{3}+p_{2} \lambda^{2}+p_{1} \lambda+p_{0}+\left(q_{1} \lambda+q_{0}\right) e^{-\lambda \tau_{1}}+\left(r_{1} \lambda+r_{0}\right) e^{-\lambda \tau_{2}}=0,
$$


where

$$
\begin{aligned}
& p_{2}=-\left(b_{11}+b_{22}+b_{33}\right), \\
& p_{1}=b_{11} b_{22}+b_{22} b_{33}+b_{11} b_{33}, \\
& p_{0}=-b_{11} b_{22} b_{33} ; \\
& q_{1}=-b_{12} c_{21}, \quad q_{0}=b_{12} c_{21} b_{33} ; \\
& r_{1}=-b_{23} d_{32}, \quad r_{0}=b_{11} b_{23} d_{32} .
\end{aligned}
$$

We consider the following cases.

(1) $E=E_{1}$. The characteristic equation reduces to

$$
\left(\lambda-a_{1}\right)\left(\lambda+a_{2}\right)\left(\lambda+a_{3}\right)=0 .
$$

There are always a positive root $a_{1}$ and two negative roots $a_{2}, a_{3}$ of (12); hence $E_{1}$ is a saddle point.

(2) $E=E_{2}$. Equation (10) takes the form

$$
\left(\lambda+a_{1}\right)\left(\lambda+a_{2}-\frac{a_{1} a_{12}}{m_{1} a_{11}+a_{1}}\right)\left(\lambda+a_{3}\right)=0 .
$$

There is a positive root $\lambda=\left(a_{1} a_{12} /\left(m_{1} a_{11}+a_{1}\right)\right)-a_{2}$ if $a_{1} a_{12} /\left(m_{1} a_{11}+a_{1}\right)>a_{2}$; hence, $E_{2}$ is a saddle point. If $a_{1} a_{12} /\left(m_{1} a_{11}+a_{1}\right)<a_{2}, E_{2}$ is locally asymptotically stable.

(3) $E=E_{3}$. The characteristic equation is

$$
\left(\lambda-b_{33}\right)\left[\lambda^{2}-\left(b_{11}+b_{22}\right) \lambda+b_{11} b_{22}-b_{12} c_{21} e^{-\lambda \tau_{1}}\right]=0 .
$$

We will analyse the distribution of the characteristic root of (14) from Ruan and Wei [26], which is stated as follows.

Lemma 1. Consider the exponential polynomial

$$
\begin{aligned}
P( & \left.\lambda, e^{-\lambda \tau_{1}}, \ldots, e^{-\lambda \tau_{m}}\right) \\
= & \lambda^{n}+p_{1}^{(0)} \lambda^{n-1}+\cdots+p_{n-1}^{(0)} \lambda+p_{n}^{(0)} \\
& +\left[p_{1}^{(1)} \lambda^{n-1}+\cdots+p_{n-1}^{(1)} \lambda+p_{n}^{(1)}\right] e^{-\lambda \tau_{1}} \\
& +\cdots+\left[p_{1}^{(m)} \lambda^{n-1}+\cdots+p_{n-1}^{(m)} \lambda+p_{n}^{(m)}\right] e^{-\lambda \tau_{m}},
\end{aligned}
$$

where $\tau_{i} \geqslant 0(i=1,2, \ldots, m)$ and $p_{j}^{(i)}(i=0,1, \ldots, m ; j=$ $1,2, \ldots, n)$ are constants. As $\left(\tau_{1}, \tau_{2}, \ldots, \tau_{m}\right)$ vary, the sum of the order of the zeros of $P\left(\lambda, e^{-\lambda \tau_{1}}, \ldots, e^{-\lambda \tau_{m}}\right)$ on the open right half plane can change only if a zero appears on or crosses the imaginary axis.

By using Lemma 1, we can easily obtain the following results.

Lemma 2. If $E_{3}$ is a nonnegative equilibrium point, then

(1) $E_{3}$ is unstable if $b_{33}>0$;

(2) $E_{3}$ is locally asymptotically stable if $b_{33}<0, b_{11}+b_{22}<$ $0, b_{11} b_{22}-b_{12} c_{21}>0$ and $b_{11} b_{22}+b_{12} c_{21}>0$.
Proof. (1) $\lambda=b_{33}$ is a root of (14); if $b_{33}>0$, then $E_{3}$ is unstable.

(2) Clearly, $\lambda=0$ is not a root of (14); we should discuss the following equation instead of (14):

$$
\lambda^{2}-\left(b_{11}+b_{22}\right) \lambda+b_{11} b_{22}-b_{12} c_{21} e^{-\lambda \tau_{1}}=0 .
$$

Assume that $i \omega$ with $\omega>0$ is a solution of (16). Substituting $\lambda=i \omega$ into (16) and separating the real and imaginary parts yield

$$
\begin{gathered}
-\omega^{2}+b_{11} b_{22}=b_{12} c_{21} \cos \omega \tau_{1}, \\
\omega\left(b_{11}+b_{22}\right)=b_{12} c_{21} \sin \omega \tau_{1}
\end{gathered}
$$

which implies

$$
\omega^{4}+\left(b_{11}^{2}+b_{22}^{2}\right) \omega^{2}+b_{11}^{2} b_{22}^{2}-b_{12}^{2} c_{21}^{2}=0 .
$$

If $b_{11}^{2} b_{22}^{2}-b_{12}^{2} c_{21}^{2}>0$, that is $\left(b_{11} b_{22}+b_{12} c_{21}\right)\left(b_{11} b_{22}-b_{12} c_{21}\right)>$ 0 , there is no real root of (16). Hence there is no purely imaginary root of (18). When $\tau_{1}=0,(16)$ reduces to

$$
\lambda^{2}-\left(b_{11}+b_{22}\right) \lambda+b_{11} b_{22}-b_{12} c_{21}=0 .
$$

If $b_{11}+b_{22}<0$ and $b_{11} b_{22}-b_{12} c_{21}>0$, both roots of (19) have negative real parts. Thus, by using Lemma 1 , when $b_{33}<0$, $b_{11}+b_{22}<0, b_{11} b_{22}-b_{12} c_{21}>0$ and $b_{11} b_{22}+b_{12} c_{21}>0, E_{3}$ is locally asymptotically stable.

(4) $E=E_{*}$. The characteristic equation about $E_{*}$ is (10). In the following, we will analyse the distribution of roots of (10). We consider four cases.

Case a. Consider

$$
\tau_{1}=\tau_{2}=0 .
$$

The associated characteristic equation of system (3) is

$$
\lambda^{3}+p_{2} \lambda^{2}+\left(p_{1}+q_{1}+r_{1}\right) \lambda+\left(p_{0}+q_{0}+r_{0}\right)=0 .
$$

Let

$\left(H_{5}\right) p_{2}>0, p_{2}\left(p_{1}+q_{1}+r_{1}\right)-\left(p_{0}+q_{0}+r_{0}\right)>0, p_{0}+q_{0}+r_{0}>$ 0 .

By Routh-Hurwitz criterion, we have the following.

Theorem 3. For $\tau_{1}=\tau_{2}=0$, assume that $\left(H_{1}\right)-\left(H_{5}\right)$ hold. Then when $\tau_{1}=\tau_{2}=0$, the positive equilibrium $E_{*}\left(x_{1}^{*}, x_{2}^{*}, x_{3}^{*}\right)$ of system (3) is locally asymptotically stable.

Case b. Consider

$$
\tau_{1}=0, \tau_{2}>0 \text {. }
$$

The associated characteristic equation of system (3) is

$$
\lambda^{3}+p_{2} \lambda^{2}+\left(p_{1}+q_{1}\right) \lambda+\left(p_{0}+q_{0}\right)+\left(r_{1} \lambda+r_{0}\right) e^{-\lambda \tau_{2}}=0 .
$$


We want to determine if the real part of some root increases to reach zero and eventually becomes positive as $\tau$ varies. Let $\lambda=i \omega(\omega>0)$ be a root of (21); then we have

$$
\begin{aligned}
& -i \omega^{3}-p_{2} \omega^{2}+i\left(p_{1}+q_{1}\right) \omega+\left(p_{0}+q_{0}\right) \\
& +\left(r_{1} \omega i+r_{0}\right)\left(\cos \omega \tau_{2}-i \sin \omega \tau_{2}\right)=0 .
\end{aligned}
$$

Separating the real and imaginary parts, we have

$$
\begin{gathered}
-\omega^{3}+\left(p_{1}+q_{1}\right) \omega=r_{0} \sin \omega \tau_{2}-r_{1} \omega \cos \omega \tau_{2}, \\
-p_{2} \omega^{2}+\left(p_{0}+q_{0}\right)=-r_{1} \omega \sin \omega \tau_{2}-r_{0} \cos \omega \tau_{2} .
\end{gathered}
$$

It follows that

$$
\omega^{6}+m_{12} \omega^{4}+m_{11} \omega^{2}+m_{10}=0
$$

where $m_{12}=p_{2}^{2}-2\left(p_{1}+q_{1}\right), m_{11}=\left(p_{1}+q_{1}\right)^{2}-2 p_{2}\left(p_{0}+\right.$ $\left.q_{0}\right)-r_{1}^{2}, m_{10}=\left(p_{0}+q_{0}\right)^{2}-r_{0}^{2}$.

Denoting $z=\omega^{2}$, (24) becomes

$$
z^{3}+m_{12} z^{2}+m_{11} z+m_{10}=0 .
$$

Let

$$
h_{1}(z)=z^{3}+m_{12} z^{2}+m_{11} z+m_{10} ;
$$

we have

$$
\frac{d h_{1}(z)}{d z}=3 z^{2}+2 m_{12} z+m_{11}
$$

If $m_{10}=\left(p_{0}+q_{0}\right)^{2}-r_{0}^{2}<0$, then $h_{1}(0)<$ $0, \lim _{z \rightarrow+\infty} h_{1}(z)=+\infty$. We can know that (25) has at least one positive root.

If $m_{10}=\left(p_{0}+q_{0}\right)^{2}-r_{0}^{2} \geq 0$, we obtain that when $\Delta=$ $m_{12}^{2}-3 m_{11} \leq 0$, (25) has no positive roots for $z \in[0,+\infty)$. On the other hand, when $\Delta=m_{12}^{2}-3 m_{11}>0$, the following equation

$$
3 z^{2}+2 m_{12} z+m_{11}=0
$$

has two real roots: $z_{11}^{*}=\left(-m_{12}+\sqrt{\Delta}\right) / 3, z_{12}^{*}=\left(-m_{12}-\right.$ $\sqrt{\Delta}) / 3$. Because of $h_{1}^{\prime \prime}\left(z_{11}^{*}\right)=2 \sqrt{\Delta}>0, h_{1}^{\prime \prime}\left(z_{12}^{*}\right)=-2 \sqrt{\Delta}<$ $0, z_{11}^{*}$ and $z_{12}^{*}$ are the local minimum and the local maximum of $h_{1}(z)$, respectively. By the above analysis, we immediately obtain the following.

Lemma 4. (1) If $m_{10} \geq 0$ and $\Delta=m_{12}^{2}-3 m_{11} \leq 0$, (25) has no positive root for $z \in[0,+\infty)$.

(2) If $m_{10} \geq 0$ and $\Delta=m_{12}^{2}-3 m_{11}>0$, (25) has at least one positive root if and only if $z_{11}^{*}=\left(-m_{12}+\sqrt{\Delta}\right) / 3>0$ and $h_{1}\left(z_{11}^{*}\right) \leq 0$.

(3) If $m_{10}<0$, (25) has at least one positive root.

Without loss of generality, we assume that (25) has three positive roots, defined by $z_{11}, z_{12}, z_{13}$, respectively. Then (24) has three positive roots:

$$
\omega_{11}=\sqrt{z_{11}}, \quad \omega_{12}=\sqrt{z_{12}}, \quad \omega_{13}=\sqrt{z_{13}} .
$$

From (23) we have

$$
\begin{aligned}
& \cos \omega_{1 k} \tau_{2_{1 k}} \\
& \quad=\frac{r_{1} \omega_{1 k}^{4}+\left[p_{2} r_{0}-\left(q_{1}+p_{1}\right) r_{1}\right] \omega_{1 k}^{2}-r_{0}\left(q_{0}+p_{0}\right)}{r_{0}^{2}+r_{1}^{2} \omega_{1 k}^{2}} .
\end{aligned}
$$

Thus, if we denote

$$
\begin{aligned}
\tau_{2_{1 k}}^{(j)}=\frac{1}{\omega_{1 k}} & \\
\quad \times\{\arccos ( & \left(r_{1} \omega_{1 k}^{4}+\left[p_{2} r_{0}-\left(q_{1}+p_{1}\right) r_{1}\right] \omega_{1 k}^{2}\right. \\
& \left.-r_{0}\left(q_{0}+p_{0}\right)\right) \\
& \left.\left.\times\left(r_{0}^{2}+r_{1}^{2} \omega_{1 k}^{2}\right)^{-1}\right)+2 j \pi\right\},
\end{aligned}
$$

where $k=1,2,3 ; j=0,1,2, \ldots$ then $\pm i \omega_{1 k}$ is a pair of purely imaginary roots of (21) corresponding to $\tau_{2_{1 k}}^{(j)}$. Define

$$
\tau_{2_{10}}=\tau_{2_{1 k_{0}}}^{(0)}=\min _{k=1,2,3}\left\{\tau_{2_{1 k}}^{(0)}\right\}, \quad \omega_{10}=\omega_{1 k_{0}} .
$$

Let $\lambda\left(\tau_{2}\right)=\alpha\left(\tau_{2}\right)+i \omega\left(\tau_{2}\right)$ be the root of (21) near $\tau_{2}=\tau_{1_{1 k}}^{(j)}$ satisfying

$$
\alpha\left(\tau_{2_{1 k}}^{(j)}\right)=0, \quad \omega\left(\tau_{2_{1 k}}^{(j)}\right)=\omega_{1 k} .
$$

Substituting $\lambda\left(\tau_{2}\right)$ into (21) and taking the derivative with respect to $\tau_{2}$, we have

$$
\begin{aligned}
\left\{3 \lambda^{2}\right. & \left.+2 p_{2} \lambda+\left(p_{1}+q_{1}\right)+r_{1} e^{-\lambda \tau_{2}}-\tau_{2}\left(r_{1} \lambda+r_{0}\right) e^{-\lambda \tau_{2}}\right\} \frac{d \lambda}{d \tau_{2}} \\
& =\lambda\left(r_{1} \lambda+r_{0}\right) e^{-\lambda \tau_{2}} .
\end{aligned}
$$

Therefore,

$$
\begin{aligned}
{\left[\frac{d \lambda}{d \tau_{2}}\right]^{-1}=} & \frac{\left[3 \lambda^{2}+2 p_{2} \lambda+\left(p_{1}+q_{1}\right)\right] e^{\lambda \tau_{2}}}{\lambda\left(r_{1} \lambda+r_{0}\right)} \\
& +\frac{r_{1}}{\lambda\left(r_{1} \lambda+r_{0}\right)}-\frac{\tau_{2}}{\lambda} .
\end{aligned}
$$

When $\tau_{2}=\tau_{2_{1 k}}^{(j)}, \lambda\left(\tau_{2_{1 k}}^{(j)}\right)=i \omega_{1 k} \quad(k=1,2,3),\left\{\lambda\left(r_{1} \lambda+\right.\right.$ $\left.\left.r_{0}\right)\right\}\left.\right|_{\tau_{2}=\tau_{1 k}^{(j)}}=-r_{1} \omega_{1 k}^{2}+i r_{0} \omega_{1 k},\left\{\left[3 \lambda^{2}+2 p_{2} \lambda+\left(p_{1}+\right.\right.\right.$ $\left.\left.\left.q_{1}\right)\right] e^{\lambda \tau_{2}}\right\}\left.\right|_{\tau_{2}=\tau_{1 k}^{(j)}}=\left\{\left[-3 \omega_{1 k}^{2}+\left(p_{1}+q_{1}\right)\right] \cos \left(\omega_{1 k} \tau_{2_{1 k}}^{(j)}\right)-\right.$ $\left.2 p_{2} \omega_{1 k} \sin \left(\omega_{1 k} \tau_{2_{1 k}}^{(j)}\right)\right\}+i\left\{2 p_{2} \omega_{1 k} \cos \left(\omega_{1 k} \tau_{2_{1 k}}^{(j)}\right)+\left[-3 \omega_{1 k}^{2}+\left(p_{1}+\right.\right.\right.$ $\left.\left.\left.q_{1}\right)\right] \sin \left(\omega_{1 k} \tau_{2_{1 k}}^{(j)}\right)\right\}$. 
According to (35), we have

$$
\begin{aligned}
& {\left[\frac{\operatorname{Red}\left(\lambda\left(\tau_{2}\right)\right)}{d \tau_{2}}\right]_{\tau_{2}=\tau_{2_{1 k}}^{(j)}}^{-1}} \\
& =\operatorname{Re}\left[\frac{\left[3 \lambda^{2}+2 p_{2} \lambda+\left(p_{1}+q_{1}\right)\right] e^{\lambda \tau_{2}}}{\lambda\left(r_{1} \lambda+r_{0}\right)}\right]_{\tau_{2}=\tau_{21 k}^{(j)}} \\
& +\operatorname{Re}\left[\frac{r_{1}}{\lambda\left(r_{1} \lambda+r_{0}\right)}\right]_{\tau_{2}=\tau_{21 k}^{(j)}} \\
& =\frac{1}{\Lambda_{1}}\left\{-r_{1} \omega_{1 k}^{2}\left[-3 \omega_{1 k}^{2}+\left(p_{1}+q_{1}\right)\right] \cos \left(\omega_{1 k} \tau_{2_{1 k}}^{(j)}\right)\right. \\
& +2 r_{1} p_{2} \omega_{1 k}^{3} \sin \left(\omega_{1 k} \tau_{2_{1 k}}^{(j)}\right)-r_{1}^{2} \omega_{1 k}^{2} \\
& +2 r_{0} p_{2} \omega_{1 k}^{2} \cos \left(\omega_{1 k} \tau_{2_{1 k}}^{(j)}\right) \\
& \left.+r_{0}\left[-3 \omega_{1 k}^{2}+\left(p_{1}+q_{1}\right)\right] \omega_{1 k} \sin \left(\omega_{1 k} \tau_{2_{1 k}}^{(j)}\right)\right\} \\
& =\frac{1}{\Lambda_{1}}\left\{3 \omega_{1 k}^{6}+2\left[p_{2}^{2}-2\left(p_{1}+q_{1}\right)\right] \omega_{1 k}^{4}\right. \\
& \left.+\left[\left(p_{1}+q_{1}\right)^{2}-2 p_{2}\left(p_{0}+q_{0}\right)-r_{1}^{2}\right] \omega_{1 k}^{2}\right\} \\
& =\frac{1}{\Lambda_{1}}\left\{z_{1 k}\left(3 z_{1 k}^{2}+2 m_{12} z_{1 k}+m_{11}\right)\right\} \\
& =\frac{1}{\Lambda_{1}} z_{1 k} h_{1}^{\prime}\left(z_{1 k}\right) \text {, }
\end{aligned}
$$

where $\Lambda_{1}=r_{1}^{2} \omega_{1 k}^{4}+r_{0}^{2} \omega_{1 k}^{2}>0$. Notice that $\Lambda_{1}>0, z_{1 k}>0$,

$$
\begin{aligned}
& \operatorname{sign}\left\{\left[\frac{\operatorname{Re} d\left(\lambda\left(\tau_{2}\right)\right)}{d \tau_{2}}\right]_{\tau_{2}=\tau_{2_{1 k}^{(j)}}}\right\} \\
& =\operatorname{sign}\left\{\left[\frac{\operatorname{Re} d\left(\lambda\left(\tau_{2}\right)\right)}{d \tau_{2}}\right]_{\tau_{2}=\tau_{2_{1 k}(j)}}^{-1}\right\} ;
\end{aligned}
$$

then we have the following lemma.

Lemma 5. Suppose that $z_{1 k}=\omega_{1 k}^{2}$ and $h_{1}^{\prime}\left(z_{1 k}\right) \neq 0$, where $h_{1}(z)$ is defined by (26); then $d\left(\operatorname{Re} \lambda\left(\tau_{2_{1 k}}^{(j)}\right)\right) / d \tau_{2}$ has the same sign with $h_{1}^{\prime}\left(z_{1 k}\right)$.

From Lemmas 1, 4, and 5 and Theorem 3, we can easily obtain the following theorem.

Theorem 6. For $\tau_{1}=0, \tau_{2}>0$, suppose that $\left(H_{1}\right)-\left(H_{5}\right)$ hold.

(i) If $m_{10} \geq 0$ and $\Delta=m_{12}^{2}-3 m_{11} \leq 0$, then all roots of (10) have negative real parts for all $\tau_{2} \geq 0$, and the positive equilibrium $E_{*}$ is locally asymptotically stable for all $\tau_{2} \geq 0$.

(ii) If either $m_{10}<0$ or $m_{10} \geq 0, \Delta=m_{12}^{2}-3 m_{11}>$ $0, z_{11}^{*}>0$, and $h_{1}\left(z_{11}^{*}\right) \leq 0$, then $h_{1}(z)$ has at least one positive roots, and all roots of (23) have negative real parts for $\tau_{2} \in\left[0, \tau_{2_{10}}\right)$, and the positive equilibrium $E_{*}$ is locally asymptotically stable for $\tau_{2} \in\left[0, \tau_{2_{10}}\right)$.

(iii) If (ii) holds and $h_{1}^{\prime}\left(z_{1 k}\right) \neq 0$, then system (3) undergoes Hopf bifurcations at the positive equilibrium $E_{*}$ for $\tau_{2}=$ $\tau_{2_{1 k}}^{(j)},(k=1,2,3 ; j=0,1,2, \ldots)$.

Case c. Consider

$$
\tau_{1}>0, \tau_{2}=0 \text {. }
$$

The associated characteristic equation of system (3) is

$$
\lambda^{3}+p_{2} \lambda^{2}+\left(p_{1}+r_{1}\right) \lambda+\left(p_{0}+r_{0}\right)+\left(q_{1} \lambda+q_{0}\right) e^{-\lambda \tau_{1}}=0 .
$$

Similar to the analysis of Case $b$, we get the following theorem.

Theorem 7. For $\tau_{1}>0, \tau_{2}=0$, suppose that $\left(H_{1}\right)-\left(H_{5}\right)$ hold.

(i) If $m_{20} \geq 0$ and $\Delta=m_{22}^{2}-3 m_{21} \leq 0$, then all roots of (38) have negative real parts for all $\tau_{1} \geq 0$, and the positive equilibrium $E_{*}$ is locally asymptotically stable for all $\tau_{1} \geq 0$.

(ii) If either $m_{20}<0$ or $m_{20} \geq 0, \Delta=m_{22}^{2}-3 m_{21}>0$, $z_{21}^{*}>0$ and $h_{2}\left(z_{21}^{*}\right) \leq 0$, then $h_{2}(z)$ has at least one positive root $z_{2 k}$, and all roots of (38) have negative real parts for $\tau_{1} \in\left[0, \tau_{1_{20}}\right)$, and the positive equilibrium $E_{*}$ is locally asymptotically stable for $\tau_{1} \in\left[0, \tau_{1_{20}}\right)$.

(iii) If (ii) holds and $h_{2}^{\prime}\left(z_{2 k}\right) \neq 0$, then system (3) undergoes Hopf bifurcations at the positive equilibrium $E_{*}$ for $\tau_{1}=$ $\tau_{1_{2 k}}^{(j)},(k=1,2,3 ; j=0,1,2, \ldots)$,

where

$$
\begin{gathered}
m_{22}=p_{2}^{2}-2\left(p_{1}+r_{1}\right), \\
m_{21}=\left(p_{1}+r_{1}\right)^{2}-2 p_{2}\left(p_{0}+r_{0}\right)-q_{1}^{2}, \\
m_{20}=\left(p_{0}+r_{0}\right)^{2}-q_{0}^{2} ; \\
h_{2}(z)=z^{3}+m_{22} z^{2}+m_{21} z+m_{20}, \quad z_{21}^{*}=\frac{-m_{22}+\sqrt{\Delta}}{3} ; \\
\tau_{1_{2 k}(j)}=\frac{1}{\omega_{2 k}} \\
\times\left\{\operatorname { a r c c o s } \left(\left(q_{1} \omega_{2 k}^{4}+\left[p_{2} q_{0}-\left(r_{1}+p_{1}\right) q_{1}\right] \omega_{2 k}^{2}\right.\right.\right. \\
\left.\quad-q_{0}\left(r_{0}+p_{0}\right)\right) \\
\left.\left.\quad \times\left(q_{0}^{2}+q_{1}^{2} \omega_{2 k}^{2}\right)^{-1}\right)+2 j \pi\right\},
\end{gathered}
$$

where $k=1,2,3 ; j=0,1,2, \ldots$; then $\pm i \omega_{2 k}$ is a pair of purely imaginary roots of (38) corresponding to $\tau_{1_{2 k}}^{(j)}$. Define

$$
\tau_{1_{20}}=\tau_{1_{2 k_{0}}}^{(0)}=\min _{k=1,2,3}\left\{\tau_{1_{2 k}}^{(0)}\right\}, \quad \omega_{10}=\omega_{1 k_{0}} .
$$


Case d. Consider

$\tau_{1}>0, \tau_{2}>0, \tau_{1} \neq \tau_{2}$.

The associated characteristic equation of system (3) is

$$
\lambda^{3}+p_{2} \lambda^{2}+p_{1} \lambda+p_{0}+\left(q_{1} \lambda+q_{0}\right) e^{-\lambda \tau_{1}}+\left(r_{1} \lambda+r_{0}\right) e^{-\lambda \tau_{2}}=0
$$

We consider (41) with $\tau_{2}=\tau_{2}^{*}$ in its stable interval $\left[0, \tau_{2_{10}}\right)$. Regard $\tau_{1}$ as a parameter.

Let $\lambda=i \omega(\omega>0)$ be a root of $(41)$; then we have

$$
\begin{gathered}
-i \omega^{3}-p_{2} \omega^{2}+i p_{1} \omega+p_{0}+\left(i q_{1} \omega+q_{0}\right)\left(\cos \omega \tau_{1}-i \sin \omega \tau_{1}\right) \\
+\left(r_{0}+i r_{1} \omega\right)\left(\cos \omega \tau_{2}^{*}-i \sin \omega \tau_{2}^{*}\right)=0 .
\end{gathered}
$$

Separating the real and imaginary parts, we have

$$
\begin{gathered}
\omega^{3}-p_{1} \omega-r_{1} \omega \cos \omega \tau_{2}^{*}+r_{0} \sin \omega \tau_{2}^{*} \\
=q_{1} \omega \cos \omega \tau_{1}-q_{0} \sin \omega \tau_{1}, \\
p_{2} \omega^{2}-p_{0}-r_{0} \cos \omega \tau_{2}^{*}-r_{1} \omega \sin \omega \tau_{2}^{*} \\
=q_{0} \cos \omega \tau_{1}+q_{1} \omega \sin \omega \tau_{1} .
\end{gathered}
$$

It follows that

$$
\omega^{6}+m_{33} \omega^{4}+m_{32} \omega^{3}+m_{31} \omega^{2}+m_{30}=0
$$

where

$$
\begin{aligned}
& m_{33}=p_{2}^{2}-2 p_{1}-2 r_{1} \cos \omega \tau_{2}^{*}, \\
& m_{32}=2\left(r_{0}-p_{2} r_{1}\right) \sin \omega \tau_{2}^{*}, \\
& m_{31}=p_{1}^{2}-2 p_{0} p_{2}-2\left(p_{2} r_{0}-p_{1} r_{1}\right) \cos \omega \tau_{2}^{*}+r_{1}^{2}-q_{1}^{2}, \\
& m_{30}=p_{0}^{2}+2 p_{0} r_{0} \cos \omega \tau_{2}^{*}+r_{0}^{2}-q_{0}^{2} .
\end{aligned}
$$

Denote $F(\omega)=\omega^{6}+m_{33} \omega^{4}+m_{32} \omega^{3}+m_{31} \omega^{2}+m_{30}$. If $m_{30}<0$, then

$$
F(0)<0, \quad \lim _{\omega \rightarrow+\infty} F(\omega)=+\infty .
$$

We can obtain that (44) has at most six positive roots $\omega_{1}, \omega_{2}, \ldots, \omega_{6}$. For every fixed $\omega_{k}, k=1,2, \ldots, 6$, there exists a sequence $\left\{\tau_{1 k}^{(j)} \mid j=0,1,2,3, \ldots\right\}$, such that (43) holds.

Let

$$
\tau_{10}=\min \left\{\tau_{1 k}^{(j)} \mid k=1,2, \ldots, 6 ; j=0,1,2,3, \ldots\right\} .
$$

When $\tau_{1}=\tau_{1 k}^{(j)}$, (41) has a pair of purely imaginary roots $\pm i \omega_{1 k}^{(j)}$ for $\tau_{2}^{*} \in\left[0, \tau_{2_{10}}\right)$.

In the following, we assume that

$$
\left.\left(H_{6}\right)\left((d \operatorname{Re}(\lambda)) / d \tau_{1}\right)\right|_{\lambda= \pm i \omega_{1 k}^{(j)}} \neq 0 .
$$

Thus, by the general Hopf bifurcation theorem for FDEs in Hale [22], we have the following result on the stability and Hopf bifurcation in system (3).
Theorem 8. For $\tau_{1}>0, \tau_{2}>0, \tau_{1} \neq \tau_{2}$, suppose that $\left(H_{1}\right)-\left(H_{6}\right)$ is satisfied. If $m_{30}<0$ and $\tau_{2}^{*} \in\left[0, \tau_{2_{10}}\right]$, then the positive equilibrium $E_{*}$ is locally asymptotically stable for $\tau_{1} \in\left[0, \tau_{10}\right)$. System (3) undergoes Hopf bifurcations at the positive equilibrium $E_{*}$ for $\tau_{1}=\tau_{1 k}^{(j)}$.

\section{Direction and Stability of the Hopf Bifurcation}

In Section 2, we obtain the conditions under which system (3) undergoes the Hopf bifurcation at the positive equilibrium $E_{*}$. In this section, we consider with $\tau_{2}=\tau_{2}^{*} \in\left[0, \tau_{2_{10}}\right)$ and regard $\tau_{1}$ as a parameter. We will derive the explicit formulas determining the direction, stability, and period of these periodic solutions bifurcating from equilibrium $E_{*}$ at the critical values $\tau_{1}$ by using the normal form and the center manifold theory developed by Hassard et al. [23]. Without loss of generality, denote any one of these critical values $\tau_{1}=$ $\tau_{1 k}^{(j)}(k=1,2, \ldots, 6 ; j=0,1,2, \ldots)$ by $\widetilde{\tau_{1}}$, at which $(43)$ has a pair of purely imaginary roots $\pm i \omega$ and system (3) undergoes Hopf bifurcation from $E_{*}$.

Throughout this section, we always assume that $\tau_{2}^{*}<\tau_{10}$. Let $u_{1}=x_{1}-x_{1}^{*}, u_{2}=x_{1}-x_{2}^{*}, u_{3}=x_{2}-x_{3}^{*}, t=\tau_{1} t$ and $\mu=\tau_{1}-\widetilde{\tau_{1}}, \mu \in \mathscr{R}$. Then $\mu=0$ is the Hopf bifurcation value of system (3). System (3) may be written as a functional differential equation in $\mathscr{C}\left([-1,0], \mathscr{R}^{3}\right)$

$$
\dot{u}(t)=L_{\mu}\left(u_{t}\right)+f\left(\mu, u_{t}\right)
$$

where $u=\left(u_{1}, u_{2}, u_{3}\right)^{T} \in \mathscr{R}^{3}$, and

$$
\begin{gathered}
L_{\mu}(\phi)=\left(\widetilde{\tau_{1}}+\mu\right) B\left[\begin{array}{l}
\phi_{1}(0) \\
\phi_{2}(0) \\
\phi_{3}(0)
\end{array}\right]+\left(\widetilde{\tau_{1}}+\mu\right) C\left[\begin{array}{l}
\phi_{1}(-1) \\
\phi_{2}(-1) \\
\phi_{3}(-1)
\end{array}\right] \\
+\left(\widetilde{\tau_{1}}+\mu\right) D\left[\begin{array}{l}
\phi_{1}\left(-\frac{\tau_{2}^{*}}{\tau_{1}}\right) \\
\phi_{2}\left(-\frac{\tau_{2}^{*}}{\tau_{1}}\right) \\
\phi_{3}\left(-\frac{\tau_{2}^{*}}{\tau_{1}}\right)
\end{array}\right] \\
f(\mu, \phi)=\left(\widetilde{\tau_{1}}+\mu\right)\left[\begin{array}{l}
f_{1} \\
f_{2} \\
f_{3}
\end{array}\right]
\end{gathered}
$$


where $\phi=\left(\phi_{1}, \phi_{2}, \phi_{3}\right)^{T} \in \mathscr{C}\left([-1,0], \mathscr{R}^{3}\right)$, and

$$
\begin{aligned}
B= & {\left[\begin{array}{ccc}
b_{11} & b_{12} & 0 \\
0 & b_{22} & b_{23} \\
0 & 0 & b_{33}
\end{array}\right], \quad C=\left[\begin{array}{ccc}
0 & 0 & 0 \\
c_{21} & 0 & 0 \\
0 & 0 & 0
\end{array}\right], } \\
D= & {\left[\begin{array}{ccc}
0 & 0 & 0 \\
0 & 0 & 0 \\
0 & d_{32} & 0
\end{array}\right], } \\
f_{1}= & -\left(a_{11}+l_{3}\right) \phi_{1}^{2}(0)-l_{1} \phi_{1}(0) \phi_{2}(0)-l_{2} \phi_{1}^{2}(0) \phi_{2}(0) \\
& -l_{5} \phi_{1}^{3}(0)-l_{4} \phi_{1}^{3}(0) \phi_{2}(0)+\cdots, \\
f_{2}= & -l_{6} \phi_{2}(0) \phi_{3}(0)-\left(l_{7}+a_{22}\right) \phi_{2}^{2}(0)+l_{1} \phi_{1}(-1) \phi_{2}(0) \\
& +l_{3} \phi_{1}^{2}(-1)-l_{8} \phi_{2}^{3}(0)-l_{9} \phi_{2}^{2}(0) \phi_{3}(0) \\
& +l_{2} \phi_{1}^{2}(-1) \phi_{2}(0)+l_{3} \phi_{1}^{3}(-1)+l_{4} \phi_{1}^{3}(-1) \phi_{2}(0) \\
& -l_{10} \phi_{2}^{3}(0) \phi_{3}(0)+\cdots, \\
l_{10}= & \frac{1}{3 !} p_{2}^{\prime \prime \prime}\left(y_{1 *}\right) . \\
l_{4}= & \frac{1}{3 !} p_{1}^{\prime \prime \prime}\left(y_{1 *}\right) y_{2 *}, \quad l_{9}=\frac{1}{2 !} p_{2}^{\prime \prime}\left(y_{1 *}\right), \quad l_{5}=\frac{1}{3 !} p_{1}^{\prime \prime \prime}\left(x_{*}\right) y_{1 *}, \\
p_{1}(x)= & \frac{1}{1+b_{1} x} p_{1}^{\prime \prime}\left(x_{*}\right), \quad l_{3}, \quad \frac{1}{2 !} p_{1}^{\prime \prime}\left(x_{*}\right) y_{1 *}, \\
& +l_{10} \phi_{2}^{3}\left(-\frac{\tau_{2}^{*}}{\tau_{1}}\right) \phi_{3}(0)+\cdots, a_{2}, \\
f_{3}= & l_{6} \phi_{2}\left(-\frac{\tau_{2}^{*}}{\tau_{1}}\right) \phi_{3}(0)+l_{7} \phi_{2}^{2}\left(-\frac{\tau_{2}}{\tau_{1}}\right)-a_{33} \phi_{3}^{2}(0) \\
& +l_{2} \phi_{2}^{2}\left(-\frac{\tau_{2}^{*}}{\tau_{1}}\right) \phi_{3}(0)+l_{8} \phi_{2}^{3}\left(-\frac{\tau_{2}}{\tau_{1}}\right)
\end{aligned}
$$

Obviously, $L_{\mu}(\phi)$ is a continuous linear function mapping $\mathscr{C}\left([-1,0], \mathscr{R}^{3}\right)$ into $\mathscr{R}^{3}$. By the Riesz representation theorem, there exists a $3 \times 3$ matrix function $\eta(\theta, \mu)(-1 \leqslant \theta \leqslant 0)$, whose elements are of bounded variation such that

$$
L_{\mu} \phi=\int_{-1}^{0} d \eta(\theta, \mu) \phi(\theta), \quad \text { for } \phi \in \mathscr{C}\left([-1,0], \mathscr{R}^{3}\right) .
$$

In fact, we can choose

$$
d \eta(\theta, \mu)=\left(\widetilde{\tau_{1}}+\mu\right)\left[B \delta(\theta)+C \delta(\theta+1)+D \delta\left(\theta+\frac{\tau_{2}^{*}}{\tau_{1}}\right)\right],
$$

where $\delta$ is Dirac-delta function. For $\phi \in \mathscr{C}\left([-1,0], \mathscr{R}^{3}\right)$, define

$$
\begin{aligned}
& A(\mu) \phi= \begin{cases}\frac{d \phi(\theta)}{d \theta}, & \theta \in[-1,0), \\
\int_{-1}^{0} d \eta(s, \mu) \phi(s), & \theta=0,\end{cases} \\
& R(\mu) \phi= \begin{cases}0, & \theta \in[-1,0), \\
f(\mu, \phi), & \theta=0 .\end{cases}
\end{aligned}
$$

Then when $\theta=0$, the system is equivalent to

$$
\dot{x}_{t}=A(\mu) x_{t}+R(\mu) x_{t},
$$

where $x_{t}(\theta)=x(t+\theta), \theta \in[-1,0]$. For $\psi \in \mathscr{C}^{1}\left([0,1],\left(\mathscr{R}^{3}\right)^{*}\right)$, define

$$
A^{*} \psi(s)= \begin{cases}-\frac{d \psi(s)}{d s}, & s \in(0,1], \\ \int_{-1}^{0} d \eta^{T}(t, 0) \psi(-t), & s=0,\end{cases}
$$

and a bilinear inner product

$$
\langle\psi(s), \phi(\theta)\rangle=\bar{\psi}(0) \phi(0)-\int_{-1}^{0} \int_{\xi=0}^{\theta} \bar{\psi}(\xi-\theta) d \eta(\theta) \phi(\xi) d \xi,
$$

where $\eta(\theta)=\eta(\theta, 0)$. Let $A=A(0)$; then $A$ and $A^{*}$ are adjoint operators. By the discussion in Section 2, we know that $\pm i \omega \widetilde{\tau_{1}}$ are eigenvalues of $A$. Thus, they are also eigenvalues of $A^{*}$. We first need to compute the eigenvector of $A$ and $A^{*}$ corresponding to $i \omega \widetilde{\tau_{1}}$ and $-i \omega \widetilde{\tau_{1}}$, respectively. Suppose that $q(\theta)=(1, \alpha, \beta)^{T} e^{i \theta \omega \widetilde{\tau_{1}}}$ is the eigenvector of $A$ corresponding to $i \omega \widetilde{\tau_{1}}$. Then $A q(\theta)=i \omega \widetilde{\tau_{1}} q(\theta)$. From the definition of $A, L_{\mu}(\phi)$ and $\eta(\theta, \mu)$, we can easily obtain $q(\theta)=$ $(1, \alpha, \beta)^{T} e^{i \theta \omega \widetilde{\tau_{1}}}$, where

$$
\alpha=\frac{i \omega-b_{11}}{b_{12}}, \quad \beta=\frac{d_{32}\left(i \omega-b_{11}\right)}{b_{12}\left(i \omega-b_{33}\right) e^{i \omega \tau_{2}^{*}}}
$$

and $q(0)=(1, \alpha, \beta)^{T}$. Similarly, let $q^{*}(s)=D\left(1, \alpha^{*}, \beta^{*}\right) e^{i s \omega \widetilde{\tau_{1}}}$ be the eigenvector of $A^{*}$ corresponding to $-i \omega \widetilde{\tau_{1}}$. By the definition of $A^{*}$, we can compute

$$
\alpha^{*}=\frac{-i \omega-b_{11}}{c_{21} e^{i \omega \tau_{1}}}, \quad \beta^{*}=\frac{b_{23}\left(-i \omega-b_{11}\right)}{c_{21}\left(i \omega-b_{33}\right) e^{i \omega \widetilde{\tau_{1}}}} .
$$

From (57), we have

$$
\begin{aligned}
& \left\langle q^{*}(s), q(\theta)\right\rangle \\
& =\bar{D}\left(1, \bar{\alpha}^{*}, \bar{\beta}^{*}\right)(1, \alpha, \beta)^{T} \\
& \quad-\int_{-1}^{0} \int_{\xi=0}^{\theta} \bar{D}\left(1, \bar{\alpha}^{*}, \bar{\beta}^{*}\right) e^{-i \omega \widetilde{\tau_{1}}(\xi-\theta)} d \eta(\theta) \\
& \quad \times(1, \alpha, \beta)^{T} e^{i \omega \widetilde{\tau}_{1} \xi} d \xi \\
& =\bar{D}\left\{1+\alpha \bar{\alpha}^{*}+\beta \bar{\beta}^{*}+c_{21} \bar{\alpha}^{*} \widetilde{\tau_{1}} e^{-i \omega \widetilde{\tau_{1}}}+d_{32} \alpha \bar{\beta}^{*} \tau_{2}^{*} e^{-i \omega \tau_{2}^{*}}\right\} .
\end{aligned}
$$


Thus, we can choose

$$
\bar{D}=\left\{1+\alpha \bar{\alpha}^{*}+\beta \bar{\beta}^{*}+c_{21} \bar{\alpha}^{*} \widetilde{\tau_{1}} e^{-i \omega \widetilde{\tau_{1}}}+d_{32} \alpha \bar{\beta}^{*} \tau_{2}^{*} e^{-i \omega \tau_{2}^{*}}\right\}^{-1},
$$

such that $\left\langle q^{*}(s), q(\theta)\right\rangle=1,\left\langle q^{*}(s), \bar{q}(\theta)\right\rangle=0$.

In the remainder of this section, we follow the ideas in Hassard et al. [23] and use the same notations as there to compute the coordinates describing the center manifold $C_{0}$ at $\mu=0$. Let $x_{t}$ be the solution of (48) when $\mu=0$. Define

$$
z(t)=\left\langle q^{*}, x_{t}\right\rangle, \quad W(t, \theta)=x_{t}(\theta)-2 \operatorname{Re}\{z(t) q(\theta)\}
$$

On the center manifold $C_{0}$, we have

$$
\begin{aligned}
W(t, \theta)= & W(z(t), \bar{z}(t), \theta) \\
= & W_{20}(\theta) \frac{z^{2}}{2}+W_{11}(\theta) z \bar{z}+W_{02}(\theta) \frac{\bar{z}^{2}}{2} \\
& +W_{30}(\theta) \frac{z^{3}}{6}+\cdots,
\end{aligned}
$$

where $z$ and $\bar{z}$ are local coordinates for center manifold $C_{0}$ in the direction of $q$ and $\bar{q}$. Note that $W$ is real if $x_{t}$ is real. We consider only real solutions. For the solution $x_{t} \in C_{0}$ of (48), since $\mu=0$, we have

$$
\begin{aligned}
\dot{z}= & i \omega \widetilde{\tau_{1}} z+\left\langle q^{*}(\theta), f(0, W(z(t), \bar{z}(t), \theta)\right. \\
& +2 \operatorname{Re}\{z(t) q(\theta)\})\rangle \\
= & i \omega \widetilde{\tau_{1}} z+\bar{q}^{*}(0) f(0, W(z(t), \bar{z}(t), 0)+2 \operatorname{Re}\{z(t) q(0)\}) \\
= & i \omega \widetilde{\tau_{1}} z+\bar{q}^{*}(0) f_{0}(z, \bar{z}) \triangleq i \omega \widetilde{\tau_{1}} z+g(z, \bar{z}),
\end{aligned}
$$

where

$$
\begin{aligned}
g(z, \bar{z})= & \bar{q}^{*}(0) f_{0}(z, \bar{z})=g_{20} \frac{z^{2}}{2}+g_{11} z \bar{z} \\
& +g_{02} \frac{\bar{z}^{2}}{2}+g_{21} \frac{z^{2} \bar{z}}{2}+\cdots .
\end{aligned}
$$

By (62), we have $x_{t}(\theta)=\left(x_{1 t}(\theta), x_{2 t}(\theta), x_{3 t}(\theta)\right)^{T}=W(t, \theta)+$ $z q(\theta)+\bar{z} \bar{q}(\theta)$, and then

$$
\begin{aligned}
x_{1 t}(0)= & z \bar{z}+W_{20}^{(1)}(0) \frac{z^{2}}{2}+W_{11}^{(1)}(0) z \bar{z} \\
& +W_{02}^{(1)}(0) \frac{\bar{z}^{2}}{2}+o\left(|(z, \bar{z})|^{3}\right) \\
x_{2 t}(0)= & z \alpha+\bar{z} \bar{\alpha}+W_{20}^{(2)}(0) \frac{z^{2}}{2} \\
& +W_{11}^{(2)}(0) z \bar{z}+W_{02}^{(2)}(0) \frac{\bar{z}^{2}}{2}+o\left(|(z, \bar{z})|^{3}\right)
\end{aligned}
$$

$$
x_{3 t}(0)=z \beta+\bar{z} \bar{\beta}+W_{20}^{(3)}(0) \frac{z^{2}}{2}+W_{11}^{(3)}(0) z \bar{z}
$$

$$
+W_{02}^{(3)}(0) \frac{\bar{z}^{2}}{2}+o\left(|(z, \bar{z})|^{3}\right)
$$

$$
x_{1 t}(-1)=z e^{-i \omega \widetilde{\tau_{1}}}+\bar{z} e^{i \omega \widetilde{\tau_{1}}}+W_{20}^{(1)}(-1) \frac{z^{2}}{2}
$$

$$
+W_{11}^{(1)}(-1) z \bar{z}+W_{02}^{(1)}(-1) \frac{\bar{z}^{2}}{2}+o\left(|(z, \bar{z})|^{3}\right) \text {, }
$$

$$
\begin{aligned}
x_{2 t}(-1)= & z \alpha e^{-i \omega \widetilde{\tau_{1}}}+\bar{z} \bar{\alpha} e^{i \omega \widetilde{\tau_{1}}}+W_{20}^{(2)}(-1) \frac{z^{2}}{2} \\
& +W_{11}^{(2)}(-1) z \bar{z}+W_{02}^{(2)}(-1) \frac{\bar{z}^{2}}{2}+o\left(|(z, \bar{z})|^{3}\right),
\end{aligned}
$$

$$
x_{3 t}(-1)=z \beta e^{-i \omega \widetilde{\tau_{1}}}+\bar{z} \bar{\beta} e^{\omega \widetilde{\tau_{1}}}+W_{20}^{(3)}(-1) \frac{z^{2}}{2}
$$

$$
+W_{11}^{(3)}(-1) z \bar{z}+W_{02}^{(3)}(-1) \frac{\bar{z}^{2}}{2}+o\left(|(z, \bar{z})|^{3}\right) ;
$$

$$
\begin{aligned}
x_{1 t}\left(-\frac{\tau_{2}^{*}}{\tau_{10}}\right)= & z e^{-i \omega \tau_{2}^{*}}+\bar{z} e^{i \omega \tau_{2}^{*}}+W_{20}^{(1)}\left(-\frac{\tau_{2}^{*}}{\widetilde{\tau_{1}}}\right) \frac{z^{2}}{2} \\
& +W_{11}^{(1)}\left(-\frac{\tau_{2}^{*}}{\widetilde{\tau_{1}}}\right) z \bar{z}+W_{02}^{(1)}\left(-\frac{\tau_{2}^{*}}{\widetilde{\tau_{1}}}\right) \frac{\bar{z}^{2}}{2} \\
& +o\left(|(z, \bar{z})|^{3}\right),
\end{aligned}
$$

$$
\begin{aligned}
x_{2 t}\left(-\frac{\tau_{2}^{*}}{\widetilde{\tau_{1}}}\right)= & z \alpha e^{-i \omega \tau_{2}^{*}}+\bar{z} \bar{\alpha} e^{i \omega \tau_{2}^{*}}+W_{20}^{(2)}\left(-\frac{\tau_{2}^{*}}{\widetilde{\tau_{1}}}\right) \frac{z^{2}}{2} \\
& +W_{11}^{(2)}\left(-\frac{\tau_{2}^{*}}{\widetilde{\tau_{1}}}\right) z \bar{z}+W_{02}^{(2)}\left(-\frac{\tau_{2}^{*}}{\widetilde{\tau_{1}}}\right) \frac{\bar{z}^{2}}{2} \\
& +o\left(|(z, \bar{z})|^{3}\right),
\end{aligned}
$$

$$
\begin{aligned}
x_{3 t}\left(-\frac{\tau_{2}^{*}}{\widetilde{\tau_{1}}}\right)= & z \beta e^{-i \omega \tau_{2}^{*}}+\bar{z} \bar{\beta} e^{\omega \tau_{2}^{*}}+W_{20}^{(3)}\left(-\frac{\tau_{2}^{*}}{\widetilde{\tau_{1}}}\right) \frac{z^{2}}{2} \\
& +W_{11}^{(3)}\left(-\frac{\tau_{2}^{*}}{\widetilde{\tau_{1}}}\right) z \bar{z}+W_{02}^{(3)}\left(-\frac{\tau_{2}^{*}}{\widetilde{\tau_{1}}}\right) \frac{\bar{z}^{2}}{2} \\
& +o\left(|(z, \bar{z})|^{3}\right) .
\end{aligned}
$$


It follows together with (50) that

$$
\begin{aligned}
& g(z, \bar{z})=\overline{q^{*}}(0) f_{0}(z, \bar{z})=\bar{D} \widetilde{\tau}_{1}\left(1, \bar{\alpha}^{*}, \bar{\beta}^{*}\right)\left(\begin{array}{lll}
f_{1}^{(0)} & f_{2}^{(0)} & f_{3}^{(0)}
\end{array}\right)^{T} \\
&=\bar{D} \widetilde{\tau_{1}}\{[-\left(a_{11}+l_{3}\right) \phi_{1}^{2}(0)-l_{1} \phi_{1}(0) \phi_{2}(0) \\
&-l_{2} \phi_{1}^{2}(0) \phi_{2}(0)-l_{5} \phi_{1}^{3}(0)-l_{4} \phi_{1}^{3}(0) \phi_{2}(0) \\
&+\cdots] \\
&+ \bar{\alpha}^{*}\left[l_{6} \phi_{2}(0) \phi_{3}(0)\left(l_{7}+a_{22}\right) \phi_{2}^{2}(0)\right. \\
&+l_{1} \phi_{1}(-1) \phi_{2}(0) \\
&+l_{3} \phi_{1}^{2}(-1)-l_{8} \phi_{2}^{3}(0)-l_{9} \phi_{2}^{2}(0) \phi_{3}(0) \\
&\left.+l_{2} \phi_{1}^{2}(-1) \phi_{2}(0)+l_{3} \phi_{1}^{3}(-1)+\cdots\right] \\
&+\bar{\beta}^{*} {\left[l_{6} \phi_{2}\left(-\frac{\tau_{2}^{*}}{\widetilde{\tau_{1}}}\right) \phi_{3}(0)+l_{7} \phi_{2}^{2}\left(-\frac{\tau_{2}^{*}}{\widetilde{\tau_{1}}}\right)\right.} \\
&\left.\left.\quad-a_{33} \phi_{3}^{2}(0)+l_{8} \phi_{2}^{3}\left(-\frac{\tau_{2}^{*}}{\widetilde{\tau_{1}}}\right)+\cdots\right]\right\}
\end{aligned}
$$

Comparing the coefficients with (65), we have

$$
\begin{aligned}
& g_{20}=\bar{D} \widetilde{\tau_{1}}\{[\left.-2\left(a_{11}+l_{3}\right)-2 \alpha l_{1}\right] \\
&+ \bar{\alpha}^{*}\left[2 l_{1} \alpha e^{-i \omega \widetilde{\tau}_{1}}+2 l_{3} e^{-2 i \omega \widetilde{\tau}_{1}}\right. \\
&\left.-2 l_{6} \alpha \beta-2\left(l_{7}+a_{22}\right) \alpha^{2}\right] \\
&+\bar{\beta}^{*}[ 2 l_{6} \alpha \beta e^{-i \omega \tau_{2}^{*}}+2\left(l_{7}+a_{22}\right) \alpha^{2} e^{-2 i \omega \tau_{2}^{*}} \\
&\left.\left.-2 a_{33} \beta^{2}\right]\right\}, \\
& g_{11}=\bar{D} \widetilde{\tau_{1}}\{[\left.-2\left(a_{11}+l_{3}\right)-l_{1}(\alpha+\bar{\alpha})\right] \\
&+ \bar{\alpha}^{*}\left[l_{1}\left(\alpha e^{i \omega \widetilde{\tau}_{1}}+\bar{\alpha} e^{-i \omega \widetilde{1}_{1}}\right)-l_{6}(\bar{\alpha} \beta+\alpha \bar{\beta})\right. \\
&\left.-2\left(l_{7}+a_{22}\right) \alpha \bar{\alpha}+2 l_{3}\right] \\
&+ \bar{\beta}^{*}\left[l_{6}\left(\beta \bar{\alpha} e^{i \omega \tau_{2}^{*}}+\alpha \bar{\beta} e^{-i \omega \tau_{2}^{*}}\right)\right. \\
&\left.\left.+2 l_{7} \alpha \bar{\alpha}-a_{33} \beta \bar{\beta}\right]\right\}, \\
& g_{02}=2 \bar{D} \widetilde{\tau}_{1}\left\{\left[-2\left(a_{11}+l_{3}\right)-2 l_{1} \bar{\alpha}\right]\right. \\
&+\bar{\alpha}^{*}\left[-2 l_{6} \bar{\alpha} \bar{\beta}-2\left(l_{7}+a_{22}\right) \bar{\alpha}^{2}+2 l_{1} \bar{\alpha} e^{i \omega \tau_{1}}\right. \\
&\left.+2 l_{3} e^{2 i \omega \tau_{1}}\right] \\
&\left.+\bar{\beta}^{*}\left[2 l_{6} \bar{\alpha} \bar{\beta} e^{i \omega \tau_{2}^{*}}+2 l_{7} \bar{\alpha}^{2} e^{2 i \omega \tau_{2}^{*}}-a_{33} \bar{\beta}^{2}\right]\right\},
\end{aligned}
$$

$$
\begin{gathered}
g_{21}=\bar{D} \widetilde{\tau_{1}\{}\left[-\left(a_{11}+l_{3}\right)\left(2 W_{20}^{(1)}(0)+4 W_{11}^{(1)}(0)\right)\right. \\
-l_{1}\left(2 \alpha W_{11}^{(1)}(0)+\bar{\alpha} W_{20}^{(1)}(0)+W_{20}^{(2)}(0)\right. \\
\left.\left.+2 W_{11}^{(2)}(0)\right)\right] \\
+\bar{\alpha}^{*}\left[-l_{6}\left(2 \beta W_{11}^{(2)}(0)+\bar{\alpha} W_{20}^{(3)}(0)+\bar{\beta} W_{20}^{(2)}(0)\right.\right. \\
\left.+2 \alpha W_{11}^{(3)}(0)\right)-\left(l_{7}+a_{22}\right) \\
\times\left(4 \alpha W_{11}^{(2)}(0)+2 \bar{\alpha} W_{20}^{(2)}(0)\right) \\
+l_{1}\left(2 \alpha W_{11}^{(1)}(-1)+\bar{\alpha} W_{20}^{(1)}(-1)\right. \\
\left.+W_{20}^{(2)}(0) e^{i \omega \widetilde{\tau}_{1}}+2 W_{11}^{(2)}(0) e^{-i \omega \widetilde{\tau_{1}}}\right) \\
\left.+l_{3}\left(4 W_{11}^{(1)}(-1) e^{-i \omega \widetilde{\tau}_{1}}+2 W_{20}^{(1)}(-1) e^{i \omega \widetilde{\tau}_{1}}\right)\right] \\
+\bar{\beta}^{*}\left[l _ { 6 } \left(2 \beta W_{11}^{(2)}\left(-\frac{\tau_{2}^{*}}{\widetilde{\tau_{1}}}\right)+\bar{\beta} W_{20}^{(2)}\left(-\frac{\tau_{2}^{*}}{\widetilde{\tau_{1}}}\right)\right.\right. \\
\left.+\bar{\alpha} W_{20}^{(3)}(0) e^{i \omega \tau_{2}^{*}}+2 \alpha W_{11}^{(3)}(0) e^{-i \omega \tau_{2}^{*}}\right) \\
+l_{7}\left(4 \alpha W_{11}^{(2)}\left(-\frac{\tau_{2}^{*}}{\widetilde{\tau_{1}}}\right) e^{-i \omega \tau_{2}^{*}}\right. \\
\left.+2 \bar{\alpha} W_{20}^{(2)}\left(-\frac{\tau_{2}^{*}}{\widetilde{\tau_{1}}}\right) e^{i \omega \tau_{2}^{*}}\right) \\
\left.\left.+a_{33}\left(4 \beta W_{11}^{(3)}(0)+2 \bar{\beta} W_{20}^{(3)}(0)\right)\right]\right\}
\end{gathered}
$$

where

$$
\begin{aligned}
& W_{20}(\theta)=\frac{i g_{20}}{\omega \widetilde{\tau_{1}}} q(0) e^{i \omega \widetilde{\tau}_{1} \theta}+\frac{i \bar{g}_{02}}{3 \omega \widetilde{\tau_{1}}} \bar{q}(0) e^{-i \omega \widetilde{\tau}_{1} \theta}+E_{1} e^{2 i \omega \widetilde{\tau_{1}} \theta}, \\
& W_{11}(\theta)=-\frac{i g_{11}}{\omega \widetilde{\tau_{1}}} q(0) e^{i \omega \widetilde{\tau}_{1} \theta}+\frac{i \bar{g}_{11}}{\omega \widetilde{\tau_{1}}} \bar{q}(0) e^{-i \omega \widetilde{\tau_{1}} \theta}+E_{2}, \\
& E_{1}=2\left[\begin{array}{ccc}
2 i \omega-b_{11} & -b_{12} & 0 \\
-c_{21} e^{-2 i \omega \widetilde{\tau_{1}}} & 2 i \omega-b_{22} & -b_{23} \\
0 & -d_{32} e^{-2 i \omega \tau_{2}^{*}} & 2 i \omega-b_{33}
\end{array}\right]^{-1} \\
& \times\left[\begin{array}{cc}
-2\left(a_{11}+l_{3}\right)-2 \alpha l_{1} \\
2 l_{1} \alpha e^{-i \omega \widetilde{\tau_{1}}}+2 l_{3} e^{-2 i \omega \widetilde{\tau}_{1}}-2 l_{6} \alpha \beta-2\left(l_{7}+a_{22}\right) \alpha^{2} \\
2 l_{6} \alpha \beta e^{-i \omega \tau_{2}^{*}}+2\left(l_{7}+a_{22}\right) \alpha^{2} e^{-2 i \omega \tau_{2}^{*}}-2 a_{33} \beta^{2}
\end{array}\right],
\end{aligned}
$$




$$
\begin{aligned}
E_{2}= & 2\left[\begin{array}{ccc}
-b_{11} & -b_{12} & 0 \\
-c_{21} & -b_{22} & -b_{23} \\
0 & -d_{32} & -b_{33}
\end{array}\right]^{-1} \\
& \times\left[\begin{array}{c}
-2\left(a_{11}+l_{3}\right)-l_{1}(\alpha+\bar{\alpha}) \\
l_{1}\left(\alpha e^{i \omega \tau_{1}}+\bar{\alpha} e^{-i \omega \widetilde{\tau_{1}}}\right)-l_{6}(\bar{\alpha} \beta+\alpha \bar{\beta})-2\left(l_{7}+a_{22}\right) \alpha \bar{\alpha}+2 l_{3} \\
l_{6}\left(\beta \bar{\alpha} e^{i \omega \tau_{2}^{*}}+\alpha \bar{\beta} e^{-i \omega \tau_{2}^{*}}\right)+2 l_{7} \alpha \bar{\alpha}-a_{33} \beta \bar{\beta}
\end{array}\right] .
\end{aligned}
$$

Thus, we can determine $W_{20}(\theta)$ and $W_{11}(\theta)$. Furthermore, we can determine each $g_{i j}$ by the parameters and delay in (3). Thus, we can compute the following values:

$$
\begin{aligned}
& c_{1}(0)=\frac{i}{2 \omega \widetilde{\tau_{1}}}\left(g_{20} g_{11}-2\left|g_{11}\right|^{2}-\frac{1}{3}\left|g_{02}\right|^{2}\right)+\frac{1}{2} g_{21}, \\
& \mu_{2}=-\frac{\operatorname{Re}\left\{c_{1}(0)\right\}}{\operatorname{Re}\left\{\lambda^{\prime}\left(\widetilde{\tau_{1}}\right)\right\}}, \\
& T_{2}=-\frac{\operatorname{Im}\left\{c_{1}(0)\right\}+\mu_{2} \operatorname{Im}\left\{\lambda^{\prime}\left(\widetilde{\tau_{1}}\right)\right\}}{\omega \widetilde{\tau_{1}}}, \quad \beta_{2}=2 \operatorname{Re}\left\{c_{1}(0)\right\},
\end{aligned}
$$

which determine the quantities of bifurcating periodic solutions in the center manifold at the critical value $\widetilde{\tau_{1}}$. Suppose $\operatorname{Re}\left\{\lambda^{\prime}\left(\widetilde{\tau_{1}}\right)\right\}>0 . \mu_{2}$ determines the directions of the Hopf bifurcation: if $\mu_{2}>0(<0)$, then the Hopf bifurcation is supercritical (subcritical) and the bifurcation exists for $\tau>\widetilde{\tau_{1}}\left(<\widetilde{\tau_{1}}\right) ; \beta_{2}$ determines the stability of the bifurcation periodic solutions: the bifurcating periodic solutions are stable (unstable) if $\beta_{2}<0(>0)$; and $T_{2}$ determines the period of the bifurcating periodic solutions: the period increases (decreases) if $T_{2}>0(<0)$.

\section{Numerical Simulation}

We consider system (3) by taking the following coefficients: $a_{1}=0.3, a_{11}=5.8889, a_{12}=1, m_{1}=1, a_{2}=$ $0.1, a_{21}=27, a_{22}=12, a_{23}=12, m_{2}=1, a_{3}=$ $0.2, a_{32}=25, a_{33}=12$. We have the unique positive equilibrium $E_{*}=(0.0451,0.0357,0.0551)$.

By computation, we get $m_{10}=-0.0104, \omega_{11}=0.5164$, $z_{11}=0.2666, h_{1}^{\prime}\left(z_{11}\right)=0.3402, \tau_{2_{0}}=3.2348$. From Theorem 6, we know that when $\tau_{1}=0$, the positive equilibrium $E_{*}$ is locally asymptotically stable for $\tau_{2} \in$ $[0,3.2348)$. When $\tau_{2}$ crosses $\tau_{2_{0}}$, the equilibrium $E_{*}$ loses its stability and Hopf bifurcation occurs. From the algorithm in Section 3, we have $\mu_{2}=566.46, \beta_{2}=-315.83, T_{2}=54.45$, which means that the bifurcation is supercritical and periodic solution is stable. The trajectories and the phase graphs are shown in Figures 1 and 2.

Regarding $\tau_{1}$ as a parameter and let $\tau_{2}=2.9 \epsilon$ $[0,3.2348)$, we can observe that with $\tau_{1}$ increasing, the positive equilibrium $E_{*}$ loses its stability and Hopf bifurcation occurs (see Figures 3 and 4).

\section{Global Continuation of Local Hopf Bifurcations}

In this section, we study the global continuation of periodic solutions bifurcating from the positive equilibrium $\left(E_{*}, \tau_{1 k}^{(j)}\right),(k=1,2, \ldots, 6 ; j=0,1, \ldots)$. Throughout this section, we follow closely the notations in [24] and assume that $\tau_{2}=\tau_{2}^{*} \in\left[0, \tau_{2_{10}}\right)$ regarding $\tau_{1}$ as a parameter. For simplification of notations, setting $z_{t}(t)=\left(x_{1 t}, x_{2 t}, x_{3 t}\right)^{T}$, we may rewrite system (3) as the following functional differential equation:

$$
\dot{z}(t)=F\left(z_{t}, \tau_{1}, p\right)
$$

where $z_{t}(\theta)=\left(x_{1 t}(\theta), x_{2 t}(\theta), x_{3 t}(\theta)\right)^{T}=\left(x_{1}(t+\theta), x_{2}(t+\right.$ $\left.\theta), x_{3}(t+\theta)\right)^{T}$ for $t \geq 0$ and $\theta \in\left[-\tau_{1}, 0\right]$. Since $x_{1}(t), x_{2}(t)$, and $x_{3}(t)$ denote the densities of the prey, the predator, and the top predator, respectively; the positive solution of system (3) is of interest and its periodic solutions only arise in the first quadrant. Thus, we consider system (3) only in the domain $R_{+}^{3}=\left\{\left(x_{1}, x_{2}, x_{3}\right) \in R^{3}, x_{1}>0, x_{2}>0, x_{3}>0\right\}$. It is obvious that (71) has a unique positive equilibrium $E_{*}\left(x_{1}^{*}, x_{2}^{*}, x_{3}^{*}\right)$ in $R_{+}^{3}$ under the assumption $\left(H_{1}\right)-\left(H_{4}\right)$. Following the work of [24], we need to define

$$
\begin{aligned}
& X=C\left(\left[-\tau_{1}, 0\right], R_{+}^{3}\right), \\
& \Gamma=C l\left\{\left(z, \tau_{1}, p\right) \in \mathbf{X} \times \mathbf{R} \times \mathbf{R}^{+} ;\right. \\
& z \text { is a } p \text {-periodic solution of system (71)\}, } \\
& \mathcal{N}=\left\{\left(\bar{z}, \overline{\tau_{1}}, \bar{p}\right) ; F\left(\bar{z}, \overline{\tau_{1}}, \bar{p}\right)=0\right\} .
\end{aligned}
$$

Let $\ell_{\left(E_{*}, \tau_{1 k}^{(j)}, 2 \pi / \omega_{1 k}^{(j)}\right)}$ denote the connected component passing through $\left(E_{*}, \tau_{1 k}^{(j)}, 2 \pi / \omega_{1 k}^{(j)}\right)$ in $\Gamma$, where $\tau_{1 k}^{(j)}$ is defined by (43). We know that $\ell_{\left(E^{*}, \tau_{1 k}^{(j)}, 2 \pi / \omega_{1 k}^{(j)}\right)}$ through $\left(E_{*}, \tau_{1 k}^{(j)}, 2 \pi / \omega_{1 k}^{(j)}\right)$ is nonempty.

For the benefit of readers, we first state the global Hopf bifurcation theory due to $\mathrm{Wu}$ [24] for functional differential equations.

Lemma 9. Assume that $\left(z_{*}, \tau, p\right)$ is an isolated center satisfying the hypotheses (A1)-(A4) in [24]. Denote by $\ell_{\left(z_{*}, \tau, p\right)}$ the connected component of $\left(z_{*}, \tau, p\right)$ in $\Gamma$. Then either

(i) $\ell_{\left(z_{*}, \tau, p\right)}$ is unbounded, or

(ii) $\ell_{\left(z_{*}, \tau, p\right)}$ is bounded, $\ell_{\left(z_{*}, \tau, p\right)} \cap \Gamma$ is finite and

$$
\sum_{(z, \tau, p) \in \ell_{\left(z_{*}, \tau, p\right)} \cap \mathcal{N}} \gamma_{m}\left(z_{*}, \tau, p\right)=0,
$$

for all $m=1,2, \ldots$, where $\gamma_{m}\left(z_{*}, \tau, p\right)$ is the $m$ th crossing number of $\left(z_{*}, \tau, p\right)$ if $m \in J\left(z_{*}, \tau, p\right)$, or it is zero if otherwise.

Clearly, if (ii) in Lemma 9 is not true, then $\ell_{\left(z_{*}, \tau, p\right)}$ is unbounded. Thus, if the projections of $\ell_{\left(z_{*}, \tau, p\right)}$ onto $z$-space and onto $p$-space are bounded, then the projection of $\ell_{\left(z_{*}, \tau, p\right)}$ onto $\tau$-space is unbounded. Further, if we can show that the 

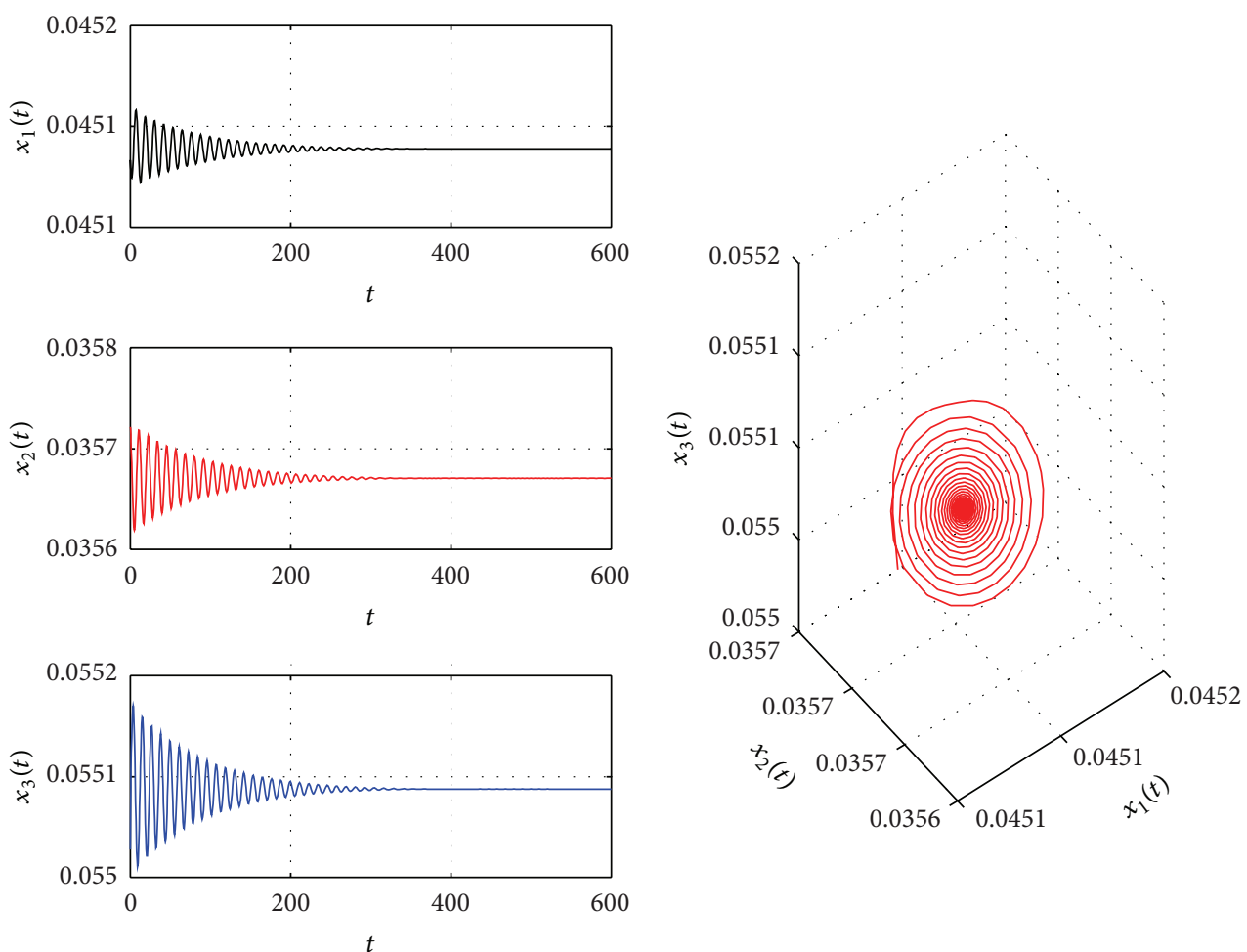

FIGURE 1: The trajectories and the phase graph with $\tau_{1}=0, \tau_{2}=2.9<\tau_{2_{0}}=3.2348 ; E_{*}$ is locally asymptotically stable.
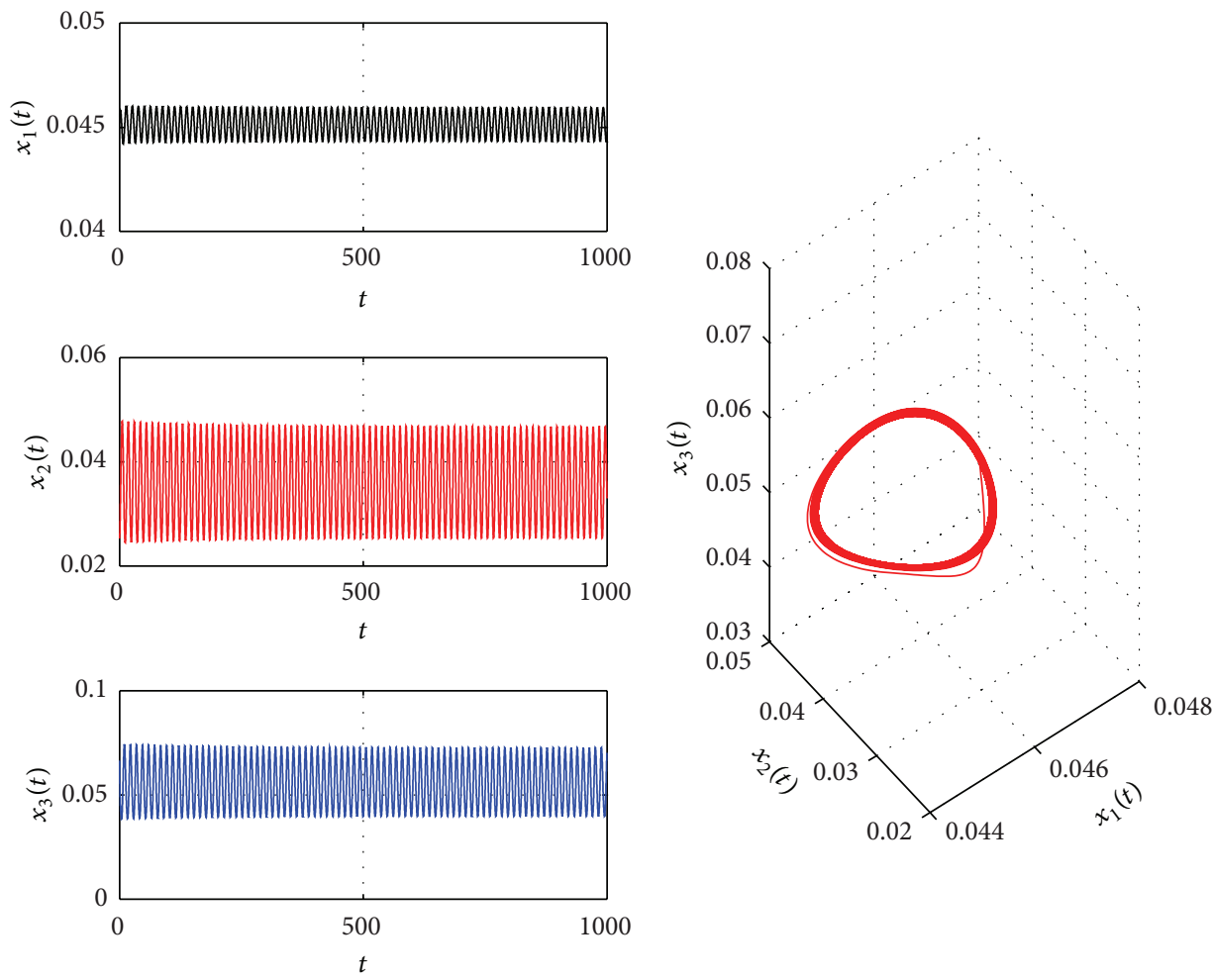

FIGURE 2: The trajectories and the phase graph with $\tau_{1}=0, \tau_{2}=3.3>\tau_{2_{0}}=3.2348$; a periodic orbit bifurcate from $E_{*}$. 

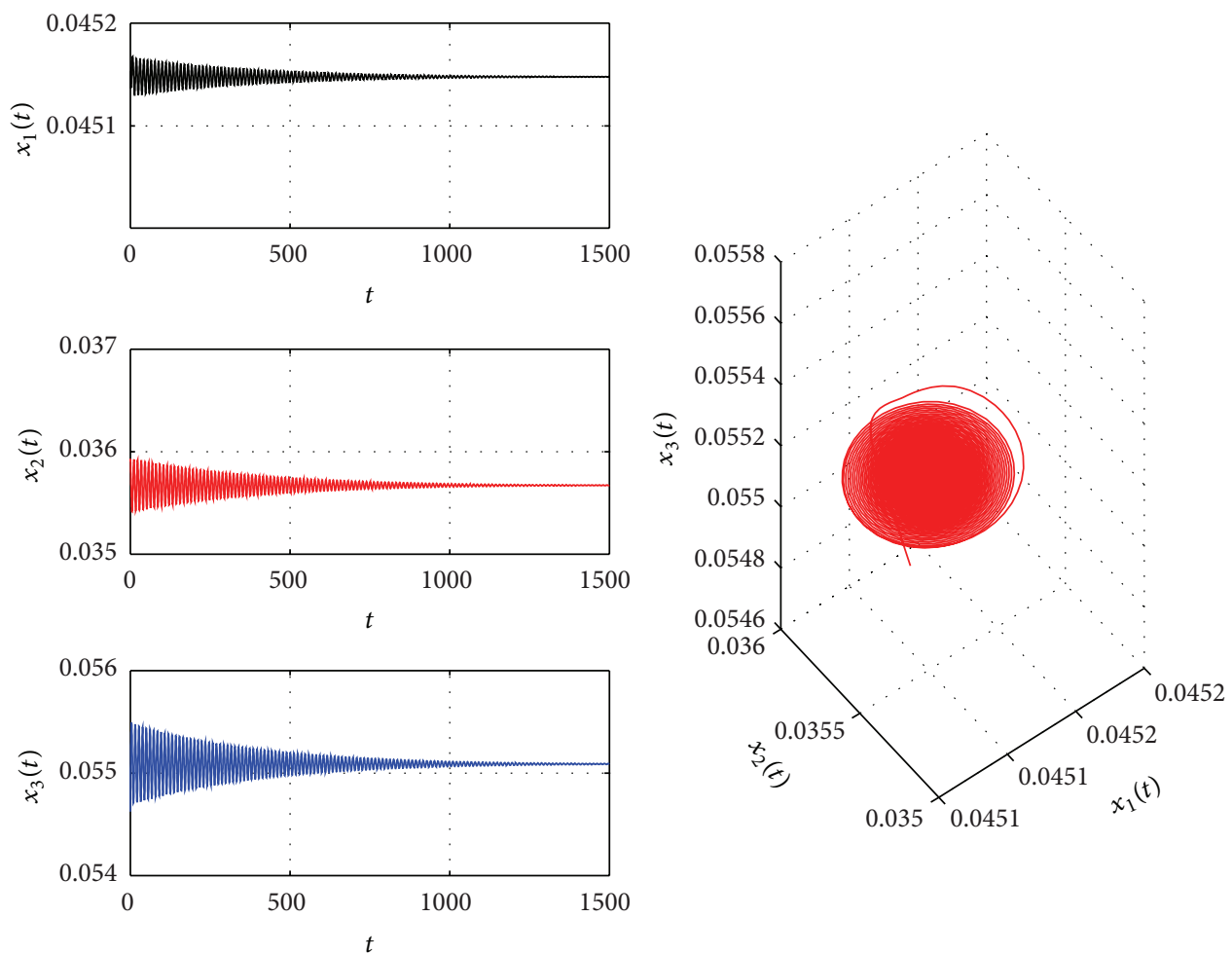

FIGURE 3: The trajectories and the phase graph with $\tau_{1}=0.9, \tau_{2}=2.9 ; E_{*}$ is locally asymptotically stable.
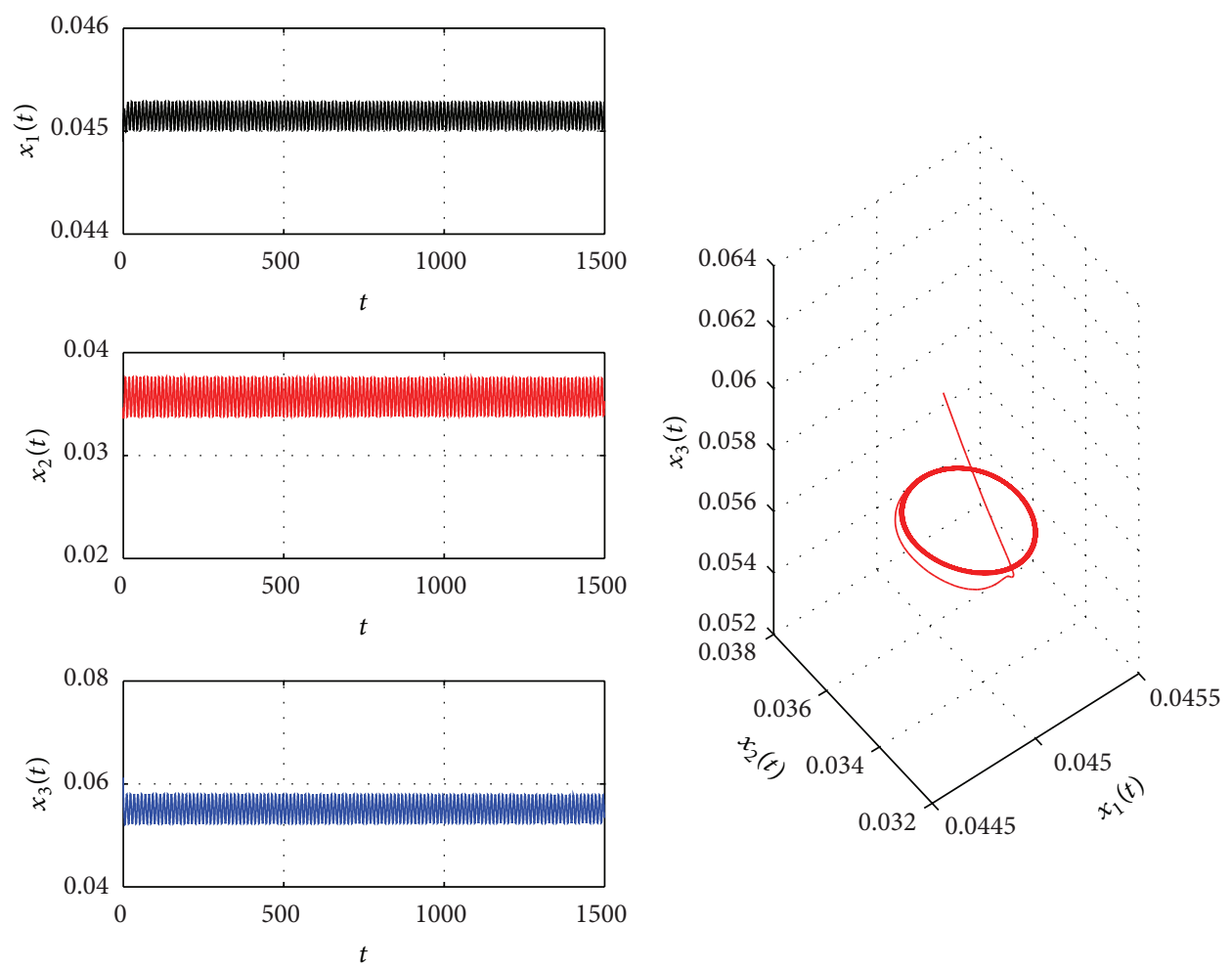

FIGURE 4: The trajectories and the phase graph with $\tau_{1}=1.2, \tau_{2}=2.9$; a periodic orbit bifurcate from $E_{*}$. 
projection of $\ell_{\left(z_{*}, \tau, p\right)}$ onto $\tau$-space is away from zero, then the projection of $\ell_{\left(z_{*}, \tau, p\right)}$ onto $\tau$-space must include interval $[\tau, \infty)$. Following this ideal, we can prove our results on the global continuation of local Hopf bifurcation.

Lemma 10. If the conditions $\left(H_{1}\right)-\left(H_{4}\right)$ hold, then all nontrivial periodic solutions of system (71) with initial conditions

$$
\begin{aligned}
& x_{1}(\theta)=\varphi(\theta) \geq 0, \quad x_{2}(\theta)=\psi(\theta) \geq 0, \\
& x_{3}(\theta)=\phi(\theta) \geq 0, \quad \theta \in\left[-\tau_{1}, 0\right) ; \\
& \varphi(0)>0, \quad \psi(0)>0, \quad \phi(0)>0
\end{aligned}
$$

are uniformly bounded.

Proof. Suppose that $\left(x_{1}(t), x_{2}(t), x_{3}(t)\right)$ are nonconstant periodic solutions of system (3) and define

$$
\begin{array}{ll}
x_{1}\left(\xi_{1}\right)=\min \left\{x_{1}(t)\right\}, & x_{1}\left(\eta_{1}\right)=\max \left\{x_{1}(t)\right\}, \\
x_{2}\left(\xi_{2}\right)=\min \left\{x_{2}(t)\right\}, & x_{2}\left(\eta_{2}\right)=\max \left\{x_{2}(t)\right\}, \\
x_{3}\left(\xi_{3}\right)=\min \left\{x_{3}(t)\right\}, & x_{3}\left(\eta_{3}\right)=\max \left\{x_{3}(t)\right\} .
\end{array}
$$

It follows from system (3) that

$$
\begin{gathered}
x_{1}(t)=x_{1}(0) \exp \left\{\int_{0}^{t}\left[a_{1}-a_{11} x_{1}(s)-\frac{a_{12} x_{2}(s)}{m_{1}+x_{1}(s)}\right] d s\right\}, \\
x_{2}(t)=x_{2}(0) \exp \left\{\int _ { 0 } ^ { t } \left[-a_{2}+\frac{a_{21} x_{1}\left(s-\tau_{1}\right)}{m_{1}+x_{1}\left(s-\tau_{1}\right)}\right.\right. \\
\left.\left.-a_{22} x_{2}(s)-\frac{a_{23} x_{3}(s)}{m_{2}+x_{2}(s)}\right] d s\right\}, \\
x_{3}(t)=x_{3}(0) \exp \left\{\int _ { 0 } ^ { t } \left[-a_{3}+\frac{a_{32} x_{2}\left(s-\tau_{2}^{*}\right)}{m_{2}+x_{2}\left(s-\tau_{2}^{*}\right)}\right.\right. \\
\left.\left.-a_{33} x_{3}(s)\right] d s\right\}
\end{gathered}
$$

which implies that the solutions of system (3) cannot cross the $x_{i}$-axis $(i=1,2,3)$. Thus, the nonconstant periodic orbits must be located in the interior of first quadrant. It follows from initial data of system (3) that $x_{1}(t)>0, x_{2}(t)>$ $0, x_{3}(t)>0$ for $t \geq 0$.

From the first equation of system (3), we can get

$$
0=a_{1}-a_{11} x_{1}\left(\eta_{1}\right)-\frac{a_{12} x_{2}\left(\eta_{1}\right)}{m_{1}+x_{1}\left(\eta_{1}\right)} \leq a_{1}-a_{11} x_{1}\left(\eta_{1}\right)
$$

thus, we have

$$
x_{1}\left(\eta_{1}\right) \leq \frac{a_{1}}{a_{11}} .
$$

From the second equation of (3), we obtain

$$
\begin{aligned}
0= & -a_{2}+\frac{a_{21} x_{1}\left(\eta_{2}-\tau_{1}\right)}{m_{1}+x_{1}\left(\eta_{2}-\tau_{1}\right)}-a_{22} x_{2}\left(\eta_{2}\right) \\
& -\frac{a_{23} x_{3}\left(\eta_{2}\right)}{m_{2}+x_{2}\left(\eta_{2}\right)} \leq-a_{2}+\frac{a_{21}\left(a_{1} / a_{11}\right)}{m_{1}+\left(a_{1} / a_{11}\right)}-a_{22} x_{2}\left(\eta_{2}\right) ;
\end{aligned}
$$

therefore, one can get

$$
x_{2}\left(\eta_{2}\right) \leq \frac{-a_{2}\left(a_{11} m_{1}+a_{1}\right)+a_{1} a_{21}}{a_{22}\left(a_{11} m_{1}+a_{1}\right)} \triangleq M_{1} .
$$

Applying the third equation of system (3), we know

$$
\begin{aligned}
0 & =-a_{3}+\frac{a_{32} x_{2}\left(\eta_{3}-\tau_{2}^{*}\right)}{m_{2}+x_{2}\left(\eta_{3}-\tau_{2}^{*}\right)}-a_{33} x_{3}\left(\eta_{3}\right) \\
& \leq-a_{3}+\frac{a_{32} M_{1}}{m_{2}+M_{1}}-a_{33} x_{3}\left(\eta_{3}\right)
\end{aligned}
$$

It follows that

$$
x_{3}\left(\eta_{3}\right) \leq \frac{-a_{3}\left(m_{2}+M_{1}\right)+a_{32} M_{1}}{a_{33}\left(m_{2}+M_{1}\right)} \triangleq M_{2} .
$$

This shows that the nontrivial periodic solution of system (3) is uniformly bounded and the proof is complete.

Lemma 11. If the conditions $\left(H_{1}\right)-\left(H_{4}\right)$ and

$$
\begin{aligned}
\left(H_{7}\right) & a_{22}-\left(a_{21} / m_{2}\right)>0,\left[\left(a_{11}-a_{1} / m_{1}\right) a_{22}-\right. \\
& \left.\left(a_{12} a_{21} / m_{1}^{2}\right)\right] a_{33}-\left(a_{11}-\left(a_{1} / m_{1}\right)\right)\left[\left(a_{21} / m_{2}\right) a_{33}+\right. \\
& \left.\left(a_{23} a_{32} / m_{2}^{2}\right)\right]>0
\end{aligned}
$$

hold, then system (3) has no nontrivial $\tau_{1}$-periodic solution.

Proof. Suppose for a contradiction that system (3) has nontrivial periodic solution with period $\tau_{1}$. Then the following system (83) of ordinary differential equations has nontrivial periodic solution:

$$
\begin{aligned}
& \frac{d x_{1}}{d t}=x_{1}(t)\left[a_{1}-a_{11} x_{1}(t)-\frac{a_{12} x_{2}(t)}{m_{1}+x_{1}(t)}\right], \\
& \frac{d x_{2}}{d t}=x_{2}(t)\left[-a_{2}+\frac{a_{21} x_{1}(t)}{m_{1}+x_{1}(t)}-a_{22} x_{2}(t)-\frac{a_{23} x_{3}(t)}{m_{2}+x_{2}(t)}\right], \\
& \frac{d x_{3}}{d t}=x_{3}(t)\left[-a_{3}+\frac{a_{32} x_{2}\left(t-\tau_{2}^{*}\right)}{m_{2}+x_{2}\left(t-\tau_{2}^{*}\right)}-a_{33} x_{3}(t)\right]
\end{aligned}
$$

which has the same equilibria to system (3); that is,

$$
\begin{array}{cl}
E_{1}=(0,0,0), & E_{2}=\left(\frac{a_{1}}{a_{11}}, 0,0\right), \\
E_{3}=\left(\tilde{x}_{1}, \tilde{x}_{2}, 0\right), & E_{*}=\left(x_{1}^{*}, x_{2}^{*}, x_{3}^{*}\right) .
\end{array}
$$

Note that $x_{i}$-axis $(i=1,2,3)$ are the invariable manifold of system (83) and the orbits of system (83) do not intersect each 
other. Thus, there are no solutions crossing the coordinate axes. On the other hand, note the fact that if system (83) has a periodic solution, then there must be the equilibrium in its interior, and that $E_{1}, E_{2}, E_{3}$ are located on the coordinate axis. Thus, we conclude that the periodic orbit of system (83) must lie in the first quadrant. If $\left(\mathrm{H}_{7}\right)$ holds, it is well known that the positive equilibrium $E_{*}$ is globally asymptotically stable in the first quadrant (see [1]). Thus, there is no periodic orbit in the first quadrant too. The above discussion means that (83) does not have any nontrivial periodic solution. It is a contradiction. Therefore, the lemma is confirmed.

Theorem 12. Suppose the conditions of Theorem 8 and $\left(H_{7}\right)$ hold; let $\omega_{k}$ and $\tau_{1 k}^{(j)}$ be defined in Section 2; then when $\tau_{1}>\tau_{1 k}^{(j)}$ system (3) has at least $j-1$ periodic solutions.
Proof. It is sufficient to prove that the projection of $\ell_{\left(E_{*}, \tau_{1 k}^{(j)}, 2 \pi / \omega_{k}\right)}$ onto $\tau_{1}$-space is $\left[\bar{\tau}_{1},+\infty\right)$ for each $j \geq 1$, where $\bar{\tau}_{1} \leq \tau_{1 k}^{(j)}$.

In following we prove that the hypotheses $(A 1)-(A 4)$ in [24] hold.

(1) From system (3) we know easily that the following conditions hold:

(A1) $\widehat{F} \in C^{2}\left(R_{+}^{3} \times R_{+} \times R_{+}\right)$, where $\widehat{F}=\left.F\right|_{R_{+}^{3} \times R_{+} \times R_{+}} \rightarrow$ $R_{+}^{3}$.

(A3) $F\left(\phi, \tau_{1}, p\right)$ is differential with respect to $\phi$.

(2) It follows from system (3) that

$$
D_{z} \hat{F}\left(z, \tau_{1}, p\right)=\left[\begin{array}{ccc}
a_{1}-2 a_{11} x_{1}-\frac{a_{12} m_{1} x_{2}}{\left(m_{1}+x_{1}\right)^{2}} & -\frac{a_{12} x_{1}}{m_{1}+x_{1}} & 0 \\
\frac{a_{21} m_{1} x_{2}}{\left(m_{1}+x_{1}\right)^{2}} & -a_{2}+\frac{a_{21} x_{1}}{m_{1}+x_{1}}-2 a_{22} x_{2}-\frac{a_{23} m_{2} x_{3}}{\left(m_{2}+x_{2}\right)^{2}} & -\frac{a_{23} x_{2}}{m_{2}+x_{2}} \\
0 & \frac{a_{32} m_{2} x_{3}}{\left(m_{2}+x_{2}\right)^{2}} & -a_{3}+\frac{a_{32} x_{2}}{m_{2}+x_{2}}-2 a_{33} x_{3}
\end{array}\right] .
$$

Then under the assumption $\left(H_{1}\right)-\left(H_{4}\right)$, we have

$$
\begin{aligned}
& \operatorname{det} D_{z} \widehat{F}\left(z^{*}, \tau_{1}, p\right) \\
& =\operatorname{det}\left[\begin{array}{ccc}
-a_{11} x_{1}^{*}+\frac{a_{12} x_{1}^{*} x_{2}^{*}}{\left(m_{1}+x_{1}^{*}\right)^{2}} & \frac{a_{12} x_{1}^{*}}{m_{1}+x_{1}^{*}} & 0 \\
\frac{a_{21} m_{1} x_{2}^{*}}{\left(m_{1}+x_{1}^{*}\right)^{2}} & -a_{22} x_{2}^{*}+\frac{a_{23} x_{2}^{*} x_{3}^{*}}{\left(m_{2}+x_{2}^{*}\right)^{2}} & -\frac{a_{23} x_{2}^{*}}{m_{2}+x_{2}^{*}} \\
0 & \frac{a_{32} m_{2} x_{3}^{*}}{\left(m_{2}+x_{2}^{*}\right)^{2}} & -a_{33} x_{3}^{*}
\end{array}\right] \\
& =-\frac{a_{2}^{2} y_{1 *} y_{2 *}}{\left(1+b_{2} y_{1 *}\right)^{3}}\left[-x_{*}+\frac{a_{1} b_{1} x_{*} y_{1 *}}{\left(1+b_{1} x_{*}\right)^{2}}\right] \neq 0 .
\end{aligned}
$$

From (86), we know that the hypothesis $(A 2)$ in [24] is satisfied.

(3) The characteristic matrix of (71) at a stationary solution $\left(\bar{z}, \tau_{0}, p_{0}\right)$ where $\bar{z}=\left(\bar{z}^{(1)}, \bar{z}^{(2)}, \bar{z}^{(3)}\right) \in R^{3}$ takes the following form:

$\Delta\left(\bar{z}, \tau_{1}, p\right)(\lambda)=\lambda I d-D_{\phi} F\left(\bar{z}, \bar{\tau}_{1}, \bar{p}\right)\left(e^{\lambda} I\right)$

that is,

$\Delta\left(\bar{z}, \tau_{1}, p\right)(\lambda)$

$$
=\left[\begin{array}{ccc}
\lambda-a_{1}+2 a_{11} \bar{z}^{(1)}+\frac{a_{12} m_{1} \bar{z}^{(2)}}{\left(m_{1}+\bar{z}^{(1)}\right)^{2}} & \frac{a_{12} \bar{z}^{(1)}}{m_{1}+\bar{z}^{(1)}} & 0 \\
-\frac{a_{21} m_{1} \bar{z}^{(2)}}{\left(m_{1}+\bar{z}^{(1)}\right)^{2}} e^{-\lambda \tau_{1}} & \lambda+a_{2}-\frac{a_{21} \bar{z}^{(1)}}{m_{1}+\bar{z}^{(1)}}+2 a_{22} \bar{z}^{(2)}+\frac{a_{23} m_{2} \bar{z}^{(3)}}{\left(m_{2}+\bar{z}^{(2)}\right)^{2}} & \frac{a_{23} \bar{z}^{(2)}}{m_{2}+\bar{z}^{(2)}} \\
0 & -\frac{a_{32} m_{2} \bar{z}^{(3)}}{\left(m_{2}+\bar{z}^{(2)}\right)^{2}} e^{-\lambda \tau_{2}^{*}} & \lambda+a_{3}-\frac{a_{32} \bar{z}^{(2)}}{m_{2}+\bar{z}^{(2)}}+2 a_{33} \bar{z}^{(3)}
\end{array}\right] .
$$


From (88), we have

$$
\begin{aligned}
\operatorname{det} & \left(\Delta\left(E_{*}, \tau_{1}, p\right)(\lambda)\right) \\
= & \lambda^{3}+p_{2} \lambda^{2}+p_{1} \lambda+p_{0}+\left[\left(r_{1}+q_{1}\right) \lambda+\left(q_{0}+r_{0}\right)\right] e^{-\lambda \tau_{1}} .
\end{aligned}
$$

Note that (89) is the same as (20); from the discussion in Section 2 about the local Hopf bifurcation, it is easy to verify that $\left(E_{*}, \tau_{1 k}^{(j)}, 2 \pi / \omega_{k}\right)$ is an isolated center, and there exist $\epsilon>$ $0, \delta>0$ and a smooth curve $\lambda:\left(\tau_{1 k}^{(j)}-\delta, \tau_{1 k}^{(j)}+\delta\right) \rightarrow \mathscr{C}$ such that $\operatorname{det}\left(\Delta\left(\lambda\left(\tau_{1}\right)\right)\right)=0,\left|\lambda\left(\tau_{1}\right)-\omega_{k}\right|<\epsilon$ for all $\tau_{1} \epsilon$ $\left[\tau_{1 k}^{(j)}-\delta, \tau_{1 k}^{(j)}+\delta\right]$ and

$$
\lambda\left(\tau_{1 k}^{(j)}\right)=\omega_{k} i,\left.\quad \frac{d \operatorname{Re} \lambda\left(\tau_{1}\right)}{d \tau_{1}}\right|_{\tau_{1}=\tau_{1 k}^{(j)}}>0 .
$$

Let

$$
\Omega_{\epsilon, 2 \pi / \omega_{k}}=\left\{(\eta, p) ; 0<\eta<\epsilon,\left|p-\frac{2 \pi}{\omega_{k}}\right|<\epsilon\right\} .
$$

It is easy to see that on $\left[\tau_{1 k}^{(j)}-\delta, \tau_{1 k}^{(j)}+\delta\right] \times \partial \Omega_{\epsilon, 2 \pi / \omega_{k}}$, $\operatorname{det}\left(\Delta\left(E_{*}, \tau_{1}, p\right)(\eta+(2 \pi / p) i)\right)=0$ if and only if, $\eta=0$, $\tau_{1}=\tau_{1 k}^{(j)}, p=2 \pi / \omega_{k}, k=1,2,3 ; j=0,1,2, \ldots$.

Therefore, the hypothesis (A4) in [24] is satisfied.

If we define

$$
\begin{aligned}
& H^{ \pm}\left(E_{*}, \tau_{1 k}^{(j)}, \frac{2 \pi}{\omega_{k}}\right)(\eta, p) \\
& \quad=\operatorname{det}\left(\Delta\left(E_{*}, \tau_{1 k}^{(j)} \pm \delta, p\right)\left(\eta+\frac{2 \pi}{p} i\right)\right),
\end{aligned}
$$

then we have the crossing number of isolated center $\left(E_{*}, \tau_{1 k}^{(j)}, 2 \pi / \omega_{k}\right)$ as follows:

$$
\begin{aligned}
\gamma\left(E_{*}, \tau_{1 k}^{(j)}, \frac{2 \pi}{\omega_{k}}\right)= & \operatorname{deg}_{B}\left(H^{-}\left(E_{*}, \tau_{1 k}^{(j)}, \frac{2 \pi}{\omega_{k}}\right), \Omega_{\epsilon, 2 \pi / \omega_{k}}\right) \\
& -\operatorname{deg}_{B}\left(H^{+}\left(E_{*}, \tau_{1 k}^{(j)}, \frac{2 \pi}{\omega_{k}}\right), \Omega_{\epsilon, 2 \pi / \omega_{k}}\right) \\
= & -1 .
\end{aligned}
$$

Thus, we have

$$
\sum_{\left(\bar{z}, \bar{\tau}_{1}, \bar{p}\right) \in \mathscr{C}_{\left(E_{*}, \tau_{1 k}^{(j)}, 2 \pi / \omega_{k}\right)}} \gamma\left(\bar{z}, \bar{\tau}_{1}, \bar{p}\right)<0,
$$

where $\left(\bar{z}, \bar{\tau}_{1}, \bar{p}\right)$ has all or parts of the form $\left(E_{*}, \tau_{1 j}^{(k)}, 2 \pi / \omega_{k}\right)(j=0,1, \ldots)$. It follows from Lemma 9 that the connected component $\ell_{\left(E_{*}, \tau_{1 k}^{(j)}, 2 \pi / \omega_{k}\right)}$ through $\left(E_{*}, \tau_{1 k}^{(j)}, 2 \pi / \omega_{k}\right)$ is unbounded for each center $\left(z_{*}, \tau_{1}, p\right),(j=0,1, \ldots)$. From the discussion in Section 2 , we have

$$
\begin{aligned}
\tau_{1 k}^{(j)}=\frac{1}{\omega_{k}} & \\
\times\{\arccos ( & \left(\left(p_{2} \omega_{k}^{2}-p_{0}\right)\left(r_{0}+q_{0}\right)\right. \\
& \left.+\omega^{2}\left(\omega^{2}-p_{1}\right)\left(r_{1}+q_{1}\right)\right) \\
& \left.\left.\times\left(\left(r_{0}+q_{0}\right)^{2}+\left(r_{1}+q_{1}\right)^{2} \omega_{k}^{2}\right)^{-1}\right)+2 j \pi\right\},
\end{aligned}
$$

where $k=1,2,3 ; j=0,1, \ldots$ Thus, one can get $2 \pi / \omega_{k} \leq \tau_{1 k}^{(j)}$ for $j \geq 1$.

Now we prove that the projection of $\ell_{\left(E_{*}, \tau_{1 k}^{(j)}, 2 \pi / \omega_{k}\right)}$ onto $\tau_{1}$-space is $\left[\bar{\tau}_{1},+\infty\right)$, where $\bar{\tau}_{1} \leq \tau_{1 k}^{(j)}$. Clearly, it follows from the proof of Lemma 11 that system (3) with $\tau_{1}=0$ has no nontrivial periodic solution. Hence, the projection of $\ell_{\left(E_{*}, \tau_{1 k}^{(j)}, 2 \pi / \omega_{k}\right)}$ onto $\tau_{1}$-space is away from zero.

For a contradiction, we suppose that the projection of $\ell_{\left(E_{*}, \tau_{1 k}^{(j)}, 2 \pi / \omega_{k}\right)}$ onto $\tau_{1}$-space is bounded; this means that the projection of $\ell_{\left(E_{*}, \tau_{1 k}^{(j)}, 2 \pi / \omega_{k}\right)}$ onto $\tau_{1}$-space is included in a interval $\left(0, \tau^{*}\right)$. Noticing $2 \pi / \omega_{k}<\tau_{1 k}^{j}$ and applying Lemma 11 we have $p<\tau^{*}$ for $\left(z(t), \tau_{1}, p\right)$ belonging to $\ell_{\left(E_{*}, \tau_{1 k}^{(j)}, 2 \pi / \omega_{k}\right)}$. This implies that the projection of $\ell_{\left(E_{*}, \tau_{1 k}^{(j)}, 2 \pi / \omega_{k}\right)}$ onto $p$ space is bounded. Then, applying Lemma 10 we get that the connected component $\ell_{\left(E_{*}, \tau_{1 k}^{(j)}, 2 \pi / \omega_{k}\right)}$ is bounded. This contradiction completes the proof.

\section{Conclusion}

In this paper, we take our attention to the stability and Hopf bifurcation analysis of a predator-prey system with MichaelisMenten type functional response and two unequal delays. We obtained some conditions for local stability and Hopf bifurcation occurring. When $\tau_{1} \neq \tau_{2}$, we derived the explicit formulas to determine the properties of periodic solutions by the normal form method and center manifold theorem. Specially, the global existence results of periodic solutions bifurcating from Hopf bifurcations are also established by using a global Hopf bifurcation result due to $\mathrm{Wu}$ [24].

\section{Conflict of Interests}

The authors declare that there is no conflict of interests regarding the publication of this paper.

\section{Acknowledgments}

The authors are grateful to thank the anonymous referees for their careful reading and constructive suggestions which lead to truly significant improvement of the paper. This research is supported by the National Natural Science Foundation of China (no. 11061016). 


\section{References}

[1] R. Xu and M. A. J. Chaplain, "Persistence and global stability in a delayed predator-prey system with Michaelis-Menten type functional response," Applied Mathematics and Computation, vol. 130, no. 2-3, pp. 441-455, 2002.

[2] Y. L. Song, Y. H. Peng, and J. J. Wei, "Bifurcations for a predatorprey system with two delays," Journal of Mathematical Analysis and Applications, vol. 337, no. 1, pp. 466-479, 2008.

[3] G.-P. Hu, W.-T. Li, and X.-P. Yan, "Hopf bifurcations in a predator-prey system with multiple delays," Chaos, Solitons \& Fractals, vol. 42, no. 2, pp. 1273-1285, 2009.

[4] M. Liao, X. Tang, and C. Xu, "Bifurcation analysis for a threespecies predator-prey system with two delays," Communications in Nonlinear Science and Numerical Simulation, vol. 17, no. 1, pp. 183-194, 2012.

[5] C. Xu and P. Li, "Dynamical analysis in a delayed predatorprey model with two delays," Discrete Dynamics in Nature and Society, vol. 2012, Article ID 652947, 22 pages, 2012.

[6] J.-F. Zhang, "Stability and bifurcation periodic solutions in a Lotka-Volterra competition system with multiple delays," Nonlinear Dynamics, vol. 70, no. 1, pp. 849-860, 2012.

[7] J. Xia, Z. Yu, and R. Yuan, "Stability and Hopf bifurcation in a symmetric Lotka-Volterra predator-prey system with delays," Electronic Journal of Differential Equations, vol. 2013, no. 9, pp. 1-16, 2013.

[8] H. Wan and J. Cui, "A malaria model with two delays," Discrete Dynamics in Nature and Society, vol. 2013, Article ID 601265, 8 pages, 2013.

[9] M. Zhao, "Hopf bifurcation analysis for a semiratio-dependent predator-prey system with two delays," Abstract and Applied Analysis, vol. 2013, Article ID 495072, 13 pages, 2013.

[10] S. Feyissa and S. Banerjee, "Delay-induced oscillatory dynamics in humoral mediated immune response with two time delays," Nonlinear Analysis: Real World Applications, vol. 14, no. 1, pp. 35-52, 2013.

[11] G. Zhang, Y. Shen, and B. Chen, "Hopf bifurcation of a predatorprey system with predator harvesting and two delays," Nonlinear Dynamics, vol. 73, no. 4, pp. 2119-2131, 2013.

[12] X.-Y. Meng, H.-F. Huo, and H. Xiang, "Hopf bifurcation in a three-species system with delays," Journal of Applied Mathematics and Computing, vol. 35, no. 1-2, pp. 635-661, 2011.

[13] J.-F. Zhang, "Global existence of bifurcated periodic solutions in a commensalism model with delays," Applied Mathematics and Computation, vol. 218, no. 23, pp. 11688-11699, 2012.

[14] Y. Wang, W. Jiang, and H. Wang, "Stability and global Hopf bifurcation in toxic phytoplankton-zooplankton model with delay and selective harvesting," Nonlinear Dynamics, vol. 73, no. 1-2, pp. 881-896, 2013.

[15] Y. Ma, "Global Hopf bifurcation in the Leslie-Gower predatorprey model with two delays," Nonlinear Analysis: Real World Applications, vol. 13, no. 1, pp. 370-375, 2012.

[16] X.-Y. Meng, H.-F. Huo, and X.-B. Zhang, "Stability and global Hopf bifurcation in a delayed food web consisting of a prey and two predators," Communications in Nonlinear Science and Numerical Simulation, vol. 16, no. 11, pp. 4335-4348, 2011.

[17] Y. Song, J. Wei, and M. Han, "Local and global Hopf bifurcation in a delayed hematopoiesis model," International Journal of Bifurcation and Chaos in Applied Sciences and Engineering, vol. 14, no. 11, pp. 3909-3919, 2004.
[18] Y. Song and J. Wei, "Local Hopf bifurcation and global periodic solutions in a delayed predator-prey system," Journal of Mathematical Analysis and Applications, vol. 301, no. 1, pp. 1-21, 2005.

[19] J. Wei and M. Y. Li, "Global existence of periodic solutions in a tri-neuron network model with delays," Physica D, vol. 198, no. 1-2, pp. 106-119, 2004.

[20] T. Zhao, Y. Kuang, and H. L. Smith, "Global existence of periodic solutions in a class of delayed Gause-type predator-prey systems," Nonlinear Analysis: Theory, Methods \& Applications, vol. 28, no. 8, pp. 1373-1394, 1997.

[21] S. J. Gao, L. S. Chen, and Z. D. Teng, "Hopf bifurcation and global stability for a delayed predator-prey system with stage structure for predator," Applied Mathematics and Computation, vol. 202, no. 2, pp. 721-729, 2008.

[22] J. Hale, Theory of Functional Differential Equations, Springer, New York, NY, USA, 1977.

[23] B. Hassard, D. Kazarinoff, and Y. Wan, Theory and Applications of Hopf Bifurcation, Cambridge University Press, Cambridge, UK, 1981.

[24] J. H. Wu, "Symmetric functional-differential equations and neural networks with memory," Transactions of the American Mathematical Society, vol. 350, no. 12, pp. 4799-4838, 1998.

[25] V. Hutson, "The existence of an equilibrium for permanent systems," The Rocky Mountain Journal of Mathematics, vol. 20, no. 4, pp. 1033-1040, 1990.

[26] S. G. Ruan and J. J. Wei, "On the zeros of transcendental functions with applications to stability of delay differential equations with two delays," Dynamics of Continuous, Discrete \& Impulsive Systems A, vol. 10, no. 6, pp. 863-874, 2003. 


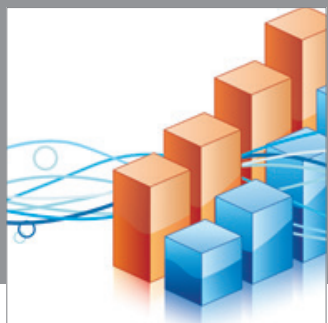

Advances in

Operations Research

mansans

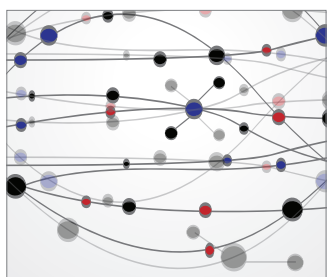

The Scientific World Journal
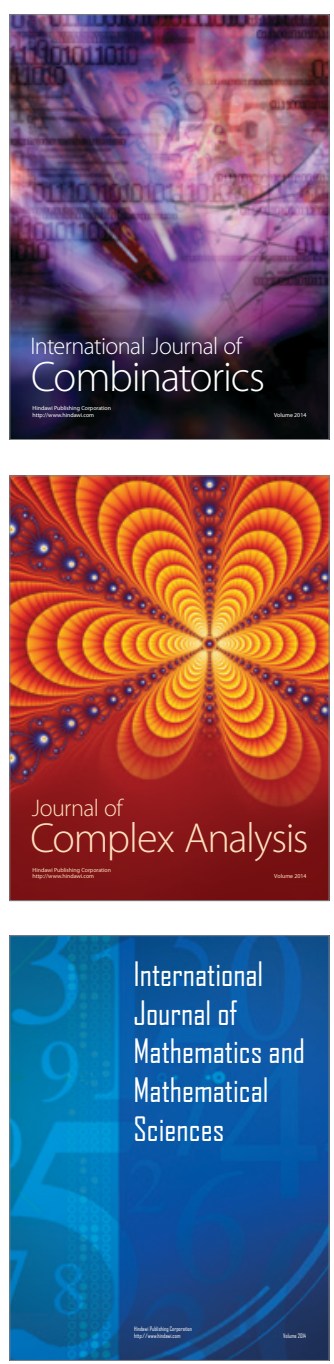
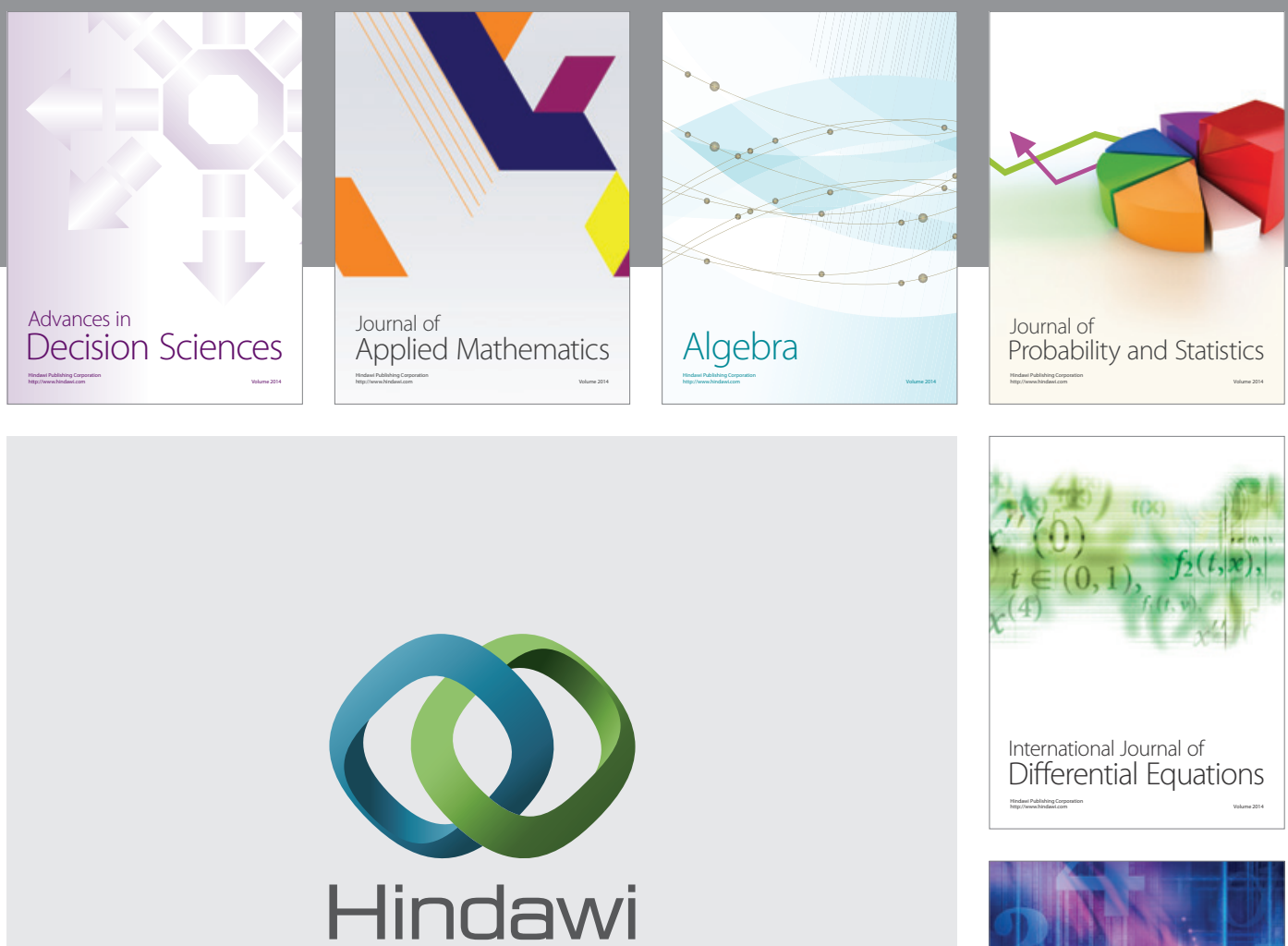

Submit your manuscripts at http://www.hindawi.com
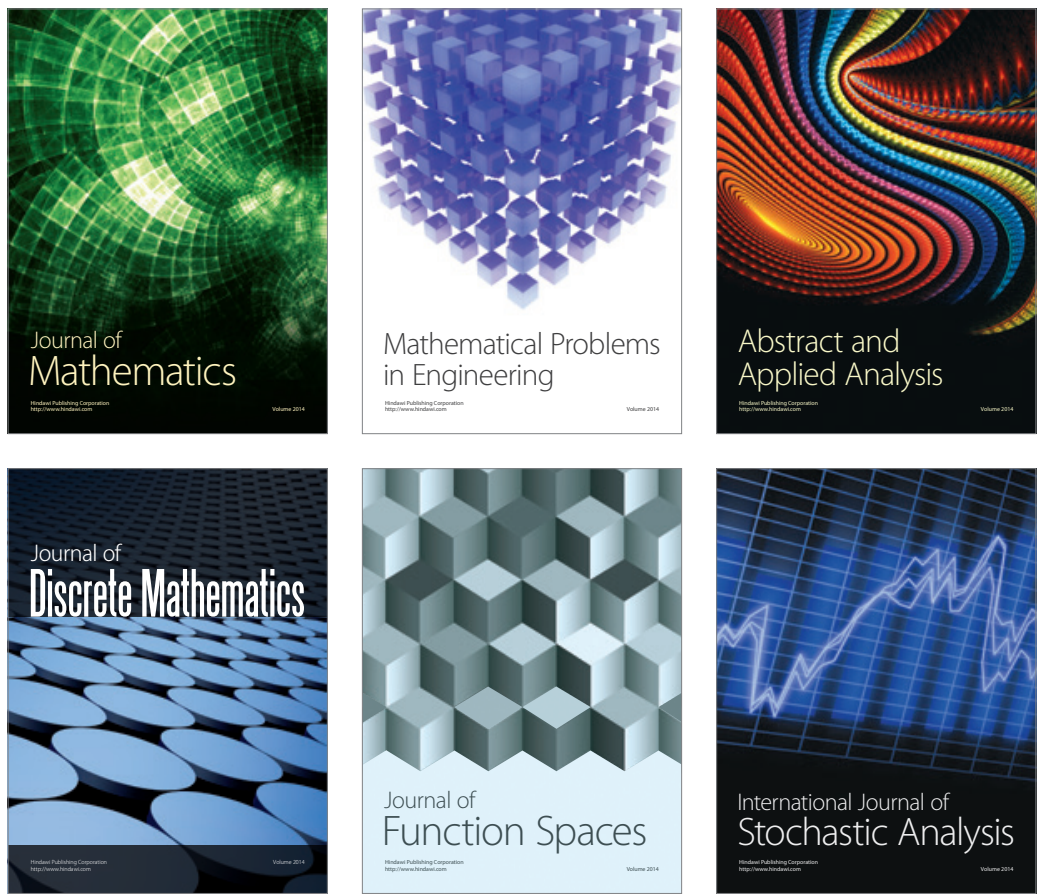

Journal of

Function Spaces

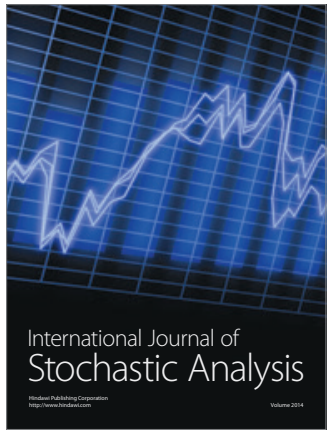

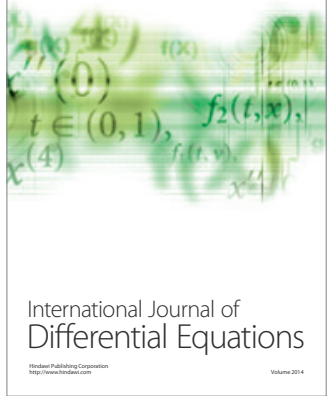
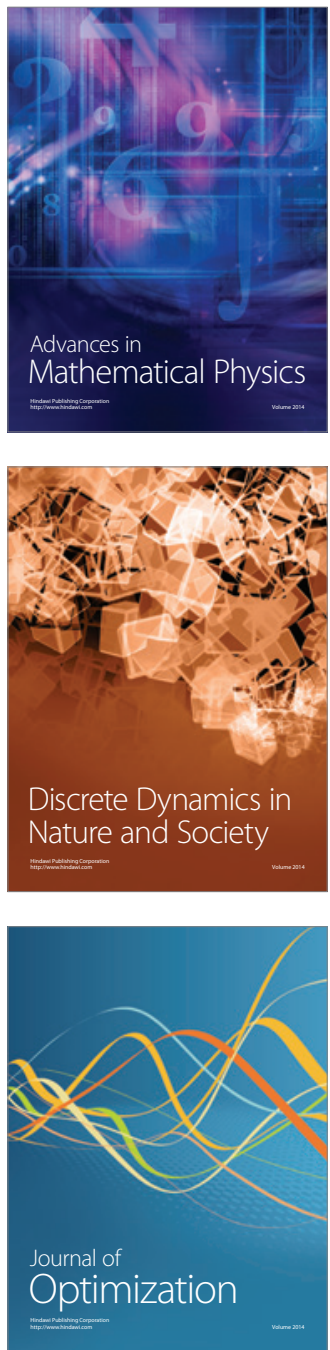